\title{
Transitions between critical kernels: from the tacnode kernel and critical kernel in the two-matrix model to the Pearcey kernel
}

\author{
Dries Geudens* and Lun Zhang*
}

August 6, 2012

\begin{abstract}
In this paper we study two multicritical correlation kernels and prove that they converge to the Pearcey kernel in a certain double scaling limit. The first kernel appears in a model of non-intersecting Brownian motions at a tacnode. The second arises as a triple scaling limit of the eigenvalue correlation kernel in the Hermitian two-matrix model with quartic/quadratic potentials. The two kernels are different but can be expressed in terms of the same tacnode Riemann-Hilbert problem. The proof is based on a steepest descent analysis of this Riemann-Hilbert problem. A special feature in the analysis is the introduction of an explicit meromorphic function on a Riemann surface with specified sheet structure.
\end{abstract}

\section{Contents}

1 Introduction 2

1.1 Non-intersecting Brownian motions at a tacnode . . . . . . . . . . 2

1.2 Hermitian two-matrix model with quartic/quadratic potentials . . . . . . 4

1.3 The tacnode Riemann-Hilbert problem . . . . . . . . . . . 5

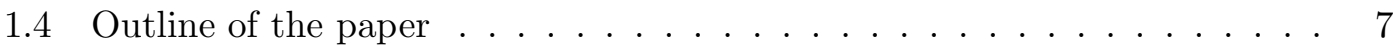

2 From the tacnode kernel to the Pearcey kernel 7

2.1 The phase diagram ..................... 7

2.2 The tacnode kernel . . . . . . . . . . . . . . . . . . . . 9

2.3 Double scaling limit: from the tacnode kernel to the Pearcey kernel . . . . 10

3 From the critical kernel to the Pearcey kernel 11

3.1 The phase diagram . . . . . . . . . . . . . . 11

3.2 The critical kernel . . . . . . . . . . . . . . . . . . . 14

3.3 Double scaling limit: from the critical kernel to the Pearcey kernel . . . . 14

\footnotetext{
${ }^{*}$ Department of Mathematics, KU Leuven, Celestijnenlaan 200B, B-3001 Leuven, Belgium. E-mail: \{dries.geudens, lun.zhang\}@wis.kuleuven.be.
} 
4 Meromorphic $\lambda$-functions on a Riemann surface 15

4.1 A four-sheeted Riemann surface and the $w$-functions . . . . . . . . . 15

4.2 The $\lambda$-functions . . . . . . . . . . . . . . . . . . 18

5 Steepest descent analysis for $M$

5.1 First transformation: $M \mapsto M^{(1)} \ldots \ldots \ldots \ldots \ldots \ldots \ldots$

5.2 Second and third transformations: $M^{(1)} \mapsto M^{(2)} \mapsto M^{(3)} \ldots \ldots \ldots \ldots .21$

5.3 Fourth transformation: $M^{(3)} \mapsto M^{(4)} \ldots \ldots \ldots \ldots \ldots \ldots$

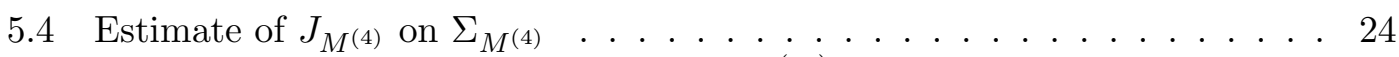

5.5 Construction of the global parametrix $M^{(\infty)} \ldots \ldots \ldots \ldots . \ldots \ldots$

5.6 Construction of the local parametrices around $\pm i c^{*} \ldots \ldots \ldots$. . . . 29

5.7 Construction of the local parametrix around the origin . . . . . . . . . 29

5.8 Final transformation: $M^{(4)} \mapsto M^{(5)} \ldots \ldots \ldots \ldots \ldots \ldots$

6 Proofs of the main theorems 36

$6.1 \mathrm{RH}$ formula for the Pearcey kernel . . . . . . . . . . . . 36

6.2 Proof of Theorem $2.3 \ldots \ldots \ldots \ldots \ldots \ldots \ldots$

6.3 Proof of Theorem $3.3 \ldots \ldots \ldots \ldots \ldots \ldots \ldots$

\section{Introduction}

Point processes with determinantal correlation kernels have attracted a lot of interest over the past few decades due to the rich mathematical structures behind them and their frequent occurrences in various random models including random matrix theory, random growth and tiling problems, etc. [34, 39]. A fundamental issue in the study of determinantal point processes is to establish microscopic limits of the correlation kernels, which often leads to universal results. Besides the well-known canonical kernels like the sine kernels for bulk universality, the Airy/Bessel kernels for soft/hard edge universality and so on, some new kernels were found describing critical behavior of certain random models, and they are believed to be universal as well. This paper deals with two such models in which a similar phenomenon occurs. We intend to retrieve a canonical process (the Pearcey process here) from the critical phenomenon. Physically, this will lead to descriptions of phase transitions among different processes. For similar transitions between canonical processes, we refer to the thesis of Deschout [25] and the recent papers [2, 6, 7].

\subsection{Non-intersecting Brownian motions at a tacnode}

As a first model we consider $n$ one-dimensional non-intersecting Brownian motions with two starting points at time $\tau=0$ and two ending points at time $\tau=1$. The transition probability density of the Brownian motions is given by

$$
P_{T}(\tau, x, y)=\frac{\sqrt{n}}{\sqrt{2 \pi \tau T}} \exp \left(-\frac{n}{2 \tau T}(x-y)^{2}\right),
$$




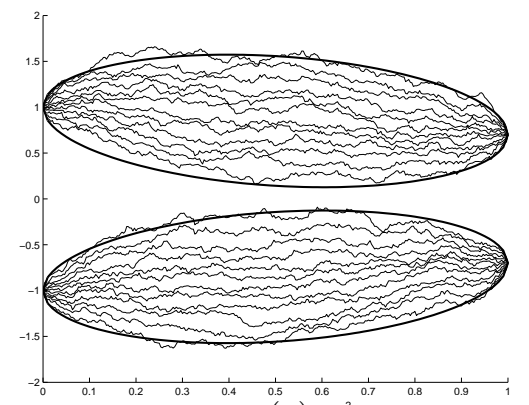

(a)

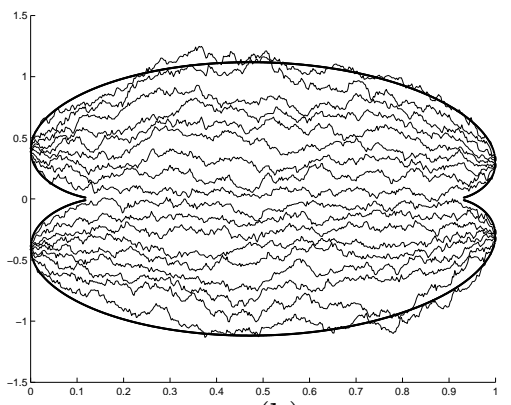

(b)

Figure 1: Non-intersecting Brownian motions with two starting points $\pm \alpha$ and two ending positions $\pm \beta$ in case of (a) large, (b) small, and (c) critical separation between the endpoints. Here the horizontal axis denotes the time, $\tau \in[0,1]$, and for each fixed $\tau$ the positions of the $n$ non-intersecting Brownian motions at time $\tau$ are denoted on the vertical line through $\tau$. Note that for $n \rightarrow \infty$ the positions of the Brownian motions fill a prescribed region in the time-space plane, which is bounded by the boldface lines in the figures. Here we have chosen $n=20, T=1$ in each of the figures, and (a) $\alpha=1$, $\beta=0.7$, (b) $\alpha=0.4, \beta=0.3$, and (c) $\alpha=1, \beta=0.5$, in the cases of large, small and critical separation, respectively.

where we interpret $T>0$ as a temperature variable. As the number of paths tends to infinity, these paths will fill out a certain domain in the time-space plane. Depending on the locations of the starting and ending points, the temperature $T$, and the fractions of paths connecting the topmost and bottommost starting and ending points, we distinguish three cases, namely, large, small, and critical separation. This is illustrated in Figure 1 which is taken from [17, see also [23].

For each fixed $\tau \in(0,1)$, the positions $x_{1}, \ldots, x_{n}$ of the Brownian paths form a determinantal point process. It is well-known that the scaling limits of the correlation kernel are given by the sine kernel in the interior of the domain, the Airy kernel at a typical point of the boundary, and the Pearcey kernel [13, 14] at a cusp [12, 40]. In the critical situation the Brownian paths fill out two touching ellipses; see Figure 1(c). The point where the ellipses touch is called the tacnode and the local particle correlations around 
this point are described by the tacnode kernel. This tacnode process was recently studied by different groups of authors using different techniques. Adler-Ferrari-Van Moerbeke [3] resolved the tacnode problem for non-intersecting random walks (discrete space and continuous time). Johansson [35] gave an integral representation of the extended tacnode kernel in the continuous time-space setting; see also Ferrari and Vetö 31] for the asymmetric tacnode process. Delvaux-Kuijlaars-Zhang [24] found an expression of the tacnode kernel in terms of a new $4 \times 4$ Riemann-Hilbert $(\mathrm{RH})$ problem. Furthermore, the tacnode process also appears in a random tiling model called the double Aztec diamond [4].

The critical separation case, see Figure 1(c), can be seen as a limit of the small separation case, in which the two groups of particles merge at a certain time and separate again later, see Figure $1(\mathrm{~b})$. In this case the particles fill out a certain shape in the timespace plane with two cusp points. At these cusp points the limiting particle density vanishes with an exponent $1 / 3$ which indicates that the local particle correlations are governed by a Pearcey kernel. When we increase the distance between both starting points and both ending points keeping the temperature $T$ fixed, or fix the starting and ending points while lowering the temperature $T$, the cusp singularities approach each other to form a tacnode. Hence we expect to retrieve the Pearcey kernel from the tacnode kernel in a certain limit. One aim of this paper is to describe such a transition.

\subsection{Hermitian two-matrix model with quartic/quadratic potentials}

A similar phenomenon occurs in the Hermitian two-matrix model, which is a probability measure

$$
\frac{1}{Z_{n}} \exp \left(-n \operatorname{Tr}\left(V\left(M_{1}\right)+W\left(M_{2}\right)-\tau M_{1} M_{2}\right)\right) \mathrm{d} M_{1} \mathrm{~d} M_{2},
$$

defined on the space of pairs $\left(M_{1}, M_{2}\right)$ of $n \times n$ Hermitian matrices. Here, $Z_{n}$ is a normalization constant, $\tau>0$ is the coupling constant, and $V$ and $W$ are two polynomials which we take as

$$
V(x)=\frac{x^{2}}{2}, \quad \text { and } \quad W(y)=\frac{y^{4}}{4}+\alpha \frac{y^{2}}{2},
$$

for a parameter $\alpha \in \mathbb{R}$.

The two-matrix model can be integrated in terms of biorthogonal polynomials, see [29, 8, 9, 10]. The correlation functions for the eigenvalues of $M_{1}$ and $M_{2}$ have a determinantal structure and admit expressions in terms of these polynomials [30, 36]. Here we are only interested in the eigenvalues of $M_{1}$ when averaged over $M_{2}$, which form a determinantal point process.

As the parameters $\alpha$ and $\tau$ vary, we encounter phase transitions. In particular, the point $(-1,1)$ in the $\alpha \tau$-plane corresponds to a multicritical case. A new kernel, referred to as the critical kernel in this paper, was recently established by Duits and Geudens [26] to describe the local eigenvalue correlations near this point. This critical kernel is expressed in terms of a $\mathrm{RH}$ problem (see $\mathrm{RH}$ problem 1.1 below) that is a generalization of the one used in the tacnode model 24]. Nevertheless, the critical kernel is genuinely different from the tacnode kernel. Although at the first glance not so clear as in the 
non-intersecting Brownian motions model, an analysis of the $\alpha \tau$-phase diagram reveals that this critical kernel can be understood as a limiting case of a Pearcey kernel or a Painlevé II kernel [11, 15]; see Section 3.1 for details. In [26] it was already shown that the critical kernel reduces to the Painlevé II kernel by taking a double scaling limit. The other aim of this paper is then to retrieve the Pearcey kernel from the critical kernel, hence, complementing the results in [26].

\subsection{The tacnode Riemann-Hilbert problem}

The essential feature allowing us to handle the tacnode kernel and the critical kernel simultaneously is that both kernels have explicit representations in terms of the following tacnode $\mathrm{RH}$ problem [24, 26].

RH problem 1.1 (Tacnode RH problem). Fix parameters $r_{1}, r_{2}, s_{1}, s_{2}$, and $t$. We look for a $4 \times 4$ matrix-valued function $M(z)$ satisfying

(1) $M$ is analytic for $z \in \mathbb{C} \backslash \Sigma_{M}$.

(2) For $z \in \Gamma_{k}$, the limiting values

$$
M_{+}(z)=\lim _{\substack{\zeta \rightarrow z \\ \zeta \text { on }+ \text {-side of } \Gamma_{k}}} M(\zeta), \quad M_{-}(z)=\lim _{\substack{\zeta \rightarrow z \\ \text { on --side of } \Gamma_{k}}} M(\zeta),
$$

exist, where the +-side and--side of $\Gamma_{k}$ are the sides which lie on the left and right of $\Gamma_{k}$, respectively, when traversing $\Gamma_{k}$ according to its orientation. These limiting values satisfy the jump relation

$$
M_{+}(z)=M_{-}(z) J_{k}(z), \quad k=0, \ldots, 9 .
$$

(3) As $z \rightarrow \infty$ with $z \in \mathbb{C} \backslash \Sigma_{M}$ we have

$$
\begin{aligned}
M(z)=\left(I+\mathcal{O}\left(z^{-1}\right)\right) & B(z) A \\
& \times \operatorname{diag}\left(e^{-\psi_{2}(z)+t z}, e^{-\psi_{1}(z)-t z}, e^{\psi_{2}(z)+t z}, e^{\psi_{1}(z)-t z}\right),
\end{aligned}
$$

where

$$
\begin{gathered}
\psi_{1}(z)=\frac{2}{3} r_{1} z^{3 / 2}+2 s_{1} z^{1 / 2}, \quad \psi_{2}(z)=\frac{2}{3} r_{2}(-z)^{3 / 2}+2 s_{2}(-z)^{1 / 2}, \\
A=\frac{1}{\sqrt{2}}\left(\begin{array}{cccc}
1 & 0 & -i & 0 \\
0 & 1 & 0 & i \\
-i & 0 & 1 & 0 \\
0 & i & 0 & 1
\end{array}\right)
\end{gathered}
$$

and

$$
B(z)=\operatorname{diag}\left((-z)^{-1 / 4}, z^{-1 / 4},(-z)^{1 / 4}, z^{1 / 4}\right) .
$$




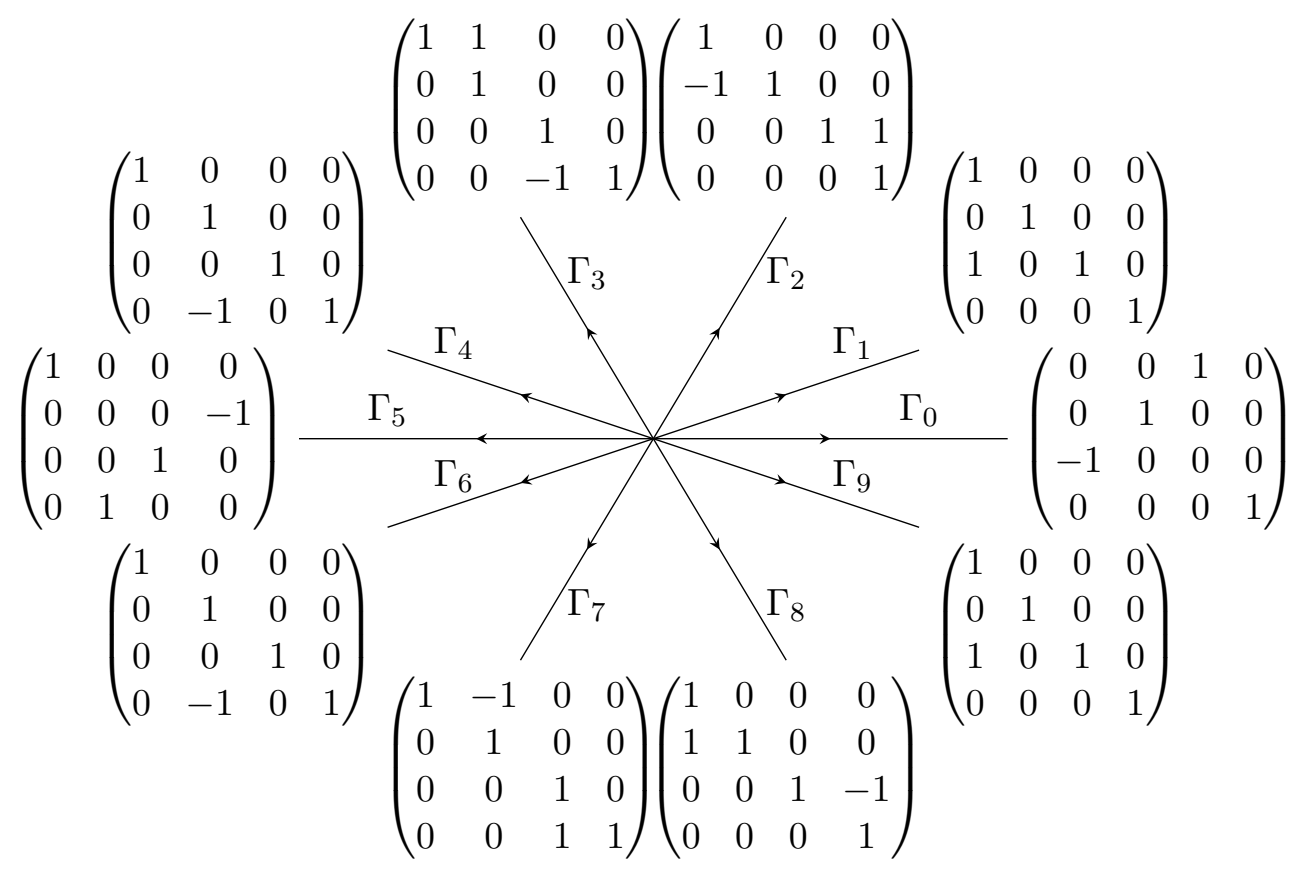

Figure 2: The jump contour $\Sigma_{M}$ in the complex $z$-plane and the constant jump matrices $J_{k}$ on each of the rays $\Gamma_{k}, k=0, \ldots, 9$.

(4) $M(z)$ is bounded near $z=0$.

The contour $\Sigma_{M}$ is shown in Figure 2 and consists of 10 rays emanating from the origin. The function $M(z)$ makes constant jumps $J_{k}$ on each of the rays $\Gamma_{k}$. These rays are determined by two angles $\varphi_{1}$ and $\varphi_{2}$ satisfying $0<\varphi_{1}<\varphi_{2}<\pi / 2$. The half-lines $\Gamma_{k}, k=0, \ldots, 9$, are defined by

$\Gamma_{0}=[0, \infty), \Gamma_{1}=\left[0, e^{i \varphi_{1}} \infty\right), \Gamma_{2}=\left[0, e^{i \varphi_{2}} \infty\right), \Gamma_{3}=\left[0, e^{i\left(\pi-\varphi_{2}\right)} \infty\right), \Gamma_{4}=\left[0, e^{i\left(\pi-\varphi_{1}\right)} \infty\right)$,

and

$$
\Gamma_{5+k}=-\Gamma_{k}, \quad k=0, \ldots, 4 .
$$

All rays are oriented towards infinity.

The fractional powers are defined with respect to the principal branch. Hence for example, $z \mapsto(-z)^{3 / 2}$ is analytic in $\mathbb{C} \backslash[0, \infty)$ and takes positive values on the negative part of the real line.

By [24, 26], this RH problem has a unique solution for $r_{1}=r_{2}>0, s_{1}=s_{2} \in \mathbb{R}$, and $t \in \mathbb{R}$. Moreover the tacnode RH problem has a remarkable connection with the Hastings-McLeod solution of the Painlevé II equation [33].

In Sections 2.2 and 3.2 below, we will see that the tacnode $\mathrm{RH}$ problem appears in 
expressions of the tacnode and critical kernel for the choices of parameters

$$
\left\{\begin{array}{l}
r_{1}=r_{2}=1 \\
s_{1}=s_{2}=s \in \mathbb{R} \\
t \in \mathbb{R}
\end{array}\right.
$$

We denote the associated solution as $M(z ; s, t)$.

\subsection{Outline of the paper}

The rest of this paper is organized as follows. Our results for the non-intersecting Brownian motions model and the Hermitian two-matrix model are stated in Sections 2 and 3, respectively. In both sections, we first discuss the model from the angle of the phase diagram, which is particularly helpful in understanding the critical phenomena and phase transitions that occur. Next we give explicit expressions of the tacnode and critical kernel in terms of the unique solution to RH problem 1.1 with parameters (1.9). Our main results are Theorems 2.3 and 3.3 , which state that the tacnode and critical kernel converge to the Pearcey kernel in a certain double scaling limit. The proofs are given in Section 6. They are based on the Deift-Zhou steepest-descent analysis [18, 19, 20] of $\mathrm{RH}$ problem 1.1, which will be performed in Section 5. A special feature in the analysis is the introduction of an explicit meromorphic $\lambda$-function on a Riemann surface with specified sheet structure, which will be the topic of Section 4. We emphasize that our approach is based on the steepest-descent analysis of a larger size RH problem as in [25] and is different from [2, 6, 7].

\section{From the tacnode kernel to the Pearcey kernel}

\subsection{The phase diagram}

We consider a symmetric version of a model of $n$ 1-dimensional non-intersecting Brownian motions as in [17, 23]. The Brownian particles start at points $\pm \alpha, \alpha>0$, at time $\tau=0$ and end at points $\pm \beta, \beta>0$, at time $\tau=1$. More precisely we assume that $n$ is even and that $n / 2$ of the particles move from the topmost starting point $\alpha$ to the topmost ending point $\beta$, while the other half of the particles connect the point $-\alpha$ to the point $-\beta$.

For convenience we fix the starting and ending points such that

$$
2 \alpha \beta=1 .
$$

According to [23], the three cases of large, small, and critical separation of the starting and ending points then correspond to $T<1, T>1$, and $T=1$, respectively; see Figure 1 again. Moreover, in case of critical separation, the coordinate of the tacnode is given by $\left(\tau_{\text {crit }}, 0\right)$, where

$$
\tau_{\text {crit }}=\frac{\alpha}{\alpha+\beta}
$$




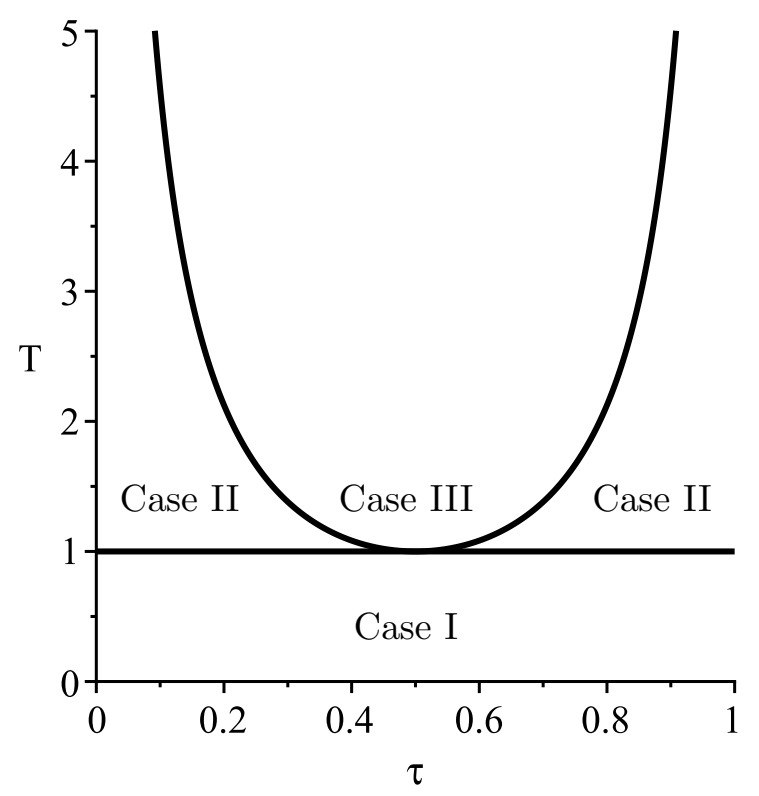

Figure 3: Phase diagram for $n$ non-intersecting Brownian motions in the symmetric setting. $n / 2$ particles connect $\alpha$ and $\beta$ while the other particles move from $-\alpha$ to $-\beta$. In this picture $\alpha=\beta=1 / \sqrt{2}$. The boldface curves indicate phase transitions and have equations $T=1$ and $T=\left(\tau^{2}-\tau+1 / 2\right) /(\tau(1-\tau))$.

is the critical time.

The assumption (2.1) allows us to summarize the situation in a clear phase diagram, shown in Figure 3 in the case $\alpha=\beta=1 / \sqrt{2}$. There are two curves in the $T \tau$-plane. One is the straight line $T=1$. The other one is given by the algebraic equation

$$
T(\tau)=\alpha^{2} \frac{1-\tau}{\tau}+\beta^{2} \frac{\tau}{1-\tau} .
$$

These two curves divide the phase plane into three regions, which we denote by Case I, Case II, and Case III. These three cases correspond to three generic situations and the two curves indicate phase transitions. Case I corresponds to temperature $T<1$, i.e., the case of large separation. In this case, the Brownian paths remain in two separate groups, and the limiting hull in the $\tau x$-plane consists of two disjoint ellipses, see Figure 1(a). The particles are asymptotically distributed on two intervals according to semicircle laws. In Cases II and III we have $T>1$ with the paths being distributed on a single interval in Case III and on two intervals in Case II. In both cases the densities of the limiting distribution for the positions of the paths at time $\tau$ are different from semicircle laws. The curve $T=1$, which corresponds to critical separation in Figure 1, separates Case I from Case II. The transition occurring at this curve was studied in [23] and is related to the homogeneous Painlevé II equation. This phase transition is not visible in the 
correlation kernel, but manifests itself in critical limits of the recurrence coefficients of the associated multiple orthogonal polynomials. The curve (2.3) separates Case II from Case III. This transition corresponds to the cusps in the limiting hull in Figure 1(b), which indicates local behavior described by Pearcey kernels.

The two critical curves touch at the multicritical point $T=1, \tau=\tau_{\text {crit }}$, which corresponds to the tacnode point in the critical separation. At this point the limiting particle density consists of two semicircles touching at the origin. In 24] the local particle correlations around the origin were studied for fixed temperature $T=1$ and time $\tau=\tau_{\text {crit }}$, i.e. exactly at the multicritical point $\left(1, \tau_{\text {crit }}\right)$, and varying starting and ending points. Under these assumptions the local scaling limit is expressed in terms of $\mathrm{RH}$ problem 1.1 with the parameter $t=0$. In this paper, however, we find it more convenient to fix the starting and ending points $\alpha$ and $\beta$ according to (2.1) and scale $(T, \tau)$ around the multicritical point as follows:

$$
\begin{aligned}
\tau & =\tau_{\text {crit }}+K n^{-1 / 3}=\frac{\alpha}{\alpha+\beta}+K n^{-1 / 3}, \\
T & =1+L n^{-2 / 3}
\end{aligned}
$$

where $K$ and $L$ are arbitrary real constants; see also [22] for a similar scaling. Then, as $n \rightarrow \infty$, the correlation kernel of non-intersection Brownian motions $K_{n}(x, y)$ at the tacnode will converge to the tacnode kernel $K^{\text {tac }}$ introduced in the next section, after proper scalings of the space variables.

\subsection{The tacnode kernel}

Definition 2.1. For $u, v \in \mathbb{R}$, we define the tacnode kernel $K^{\text {tac }}$ by

$$
K^{\mathrm{tac}}(u, v ; s, t)=\frac{1}{2 \pi i(u-v)}\left(\begin{array}{cccc}
0 & 0 & 1 & 1
\end{array}\right) \widehat{M}^{-1}(v ; s, t) \widehat{M}(u ; s, t)\left(\begin{array}{l}
1 \\
1 \\
0 \\
0
\end{array}\right),
$$

where $\widehat{M}(z ; s, t)$ denotes the analytic continuation of the restriction of $M(z ; s, t)$ to the sector around the positive imaginary axis, bounded by the rays $\Gamma_{2}$ and $\Gamma_{3}$. Recall that $M(z ; s, t)$ is the unique solution to $\mathrm{RH}$ problem 1.1 with parameters (1.9).

We can rewrite the kernel in terms of the limiting values $M_{+}(u ; s, t)$ and $M_{+}(v ; s, t)$ of $M$ on the real line, by using the jump relations in $\mathrm{RH}$ problem 1.1, For example, for $u, v>0$, we have

$$
K^{\mathrm{tac}}(u, v ; s, t)=\frac{1}{2 \pi i(u-v)}\left(\begin{array}{cccc}
-1 & 0 & 1 & 0
\end{array}\right) M_{+}^{-1}(v ; s, t) M_{+}(u ; s, t)\left(\begin{array}{l}
1 \\
0 \\
1 \\
0
\end{array}\right),
$$

with a different expression in case $u$ and/or $v$ are negative.

We then have the following results concerning the correlation kernel of non-intersecting Brownian motions at a tacnode. 
Theorem 2.2. Consider $n$ nonintersecting Brownian motions on $\mathbb{R}$ with two starting points $\pm \alpha, \alpha>0$ and two ending points $\pm \beta, \beta>0$. The transition probability density is given by (1.1). Suppose half of the paths start in $\alpha$ at time $\tau=0$ and end in $\beta$ at time $\tau=1$, while the other half of the paths connect $-\alpha$ and $-\beta$. If we fix $\alpha$ and $\beta$ such that $2 \alpha \beta=1$ and make a double scaling limit of the temperature $T$ and the time $\tau$ according to (2.4), the correlation kernel $K_{n}$ for the positions of the particles near the critical time satisfies

$$
\begin{aligned}
\lim _{n \rightarrow \infty} \frac{1}{c n^{2 / 3}} K_{n}\left(\frac{u}{c n^{2 / 3}}, \frac{v}{c n^{2 / 3}}\right) \\
\quad=K^{\operatorname{tac}}\left(u, v ; 2^{-5 / 3}\left((\alpha+\beta)^{4} K^{2}-L\right),-2^{-1 / 3}(\alpha+\beta)^{2} K\right),
\end{aligned}
$$

uniformly for $u, v$ in compact subsets of $\mathbb{R}$, where $c=2^{1 / 3}(\alpha+\beta)$ and $K^{\text {tac }}$ is given by (2.5).

Proof. A version of this theorem with different scaling assumptions was proved in [24]. The proof of this theorem follows the same lines. The main difference is in the construction of the local parametrix around the origin, which is now built out of the solution to $\mathrm{RH}$ problem 1.1] for nonzero $t$, whereas in [24] the version for $t=0$ was used. See also [22] for a similar construction in a more involved model.

\subsection{Double scaling limit: from the tacnode kernel to the Pearcey kernel}

The Pearcey kernel $K^{\mathrm{Pe}}$ is defined as (see [13, 14])

$$
K^{\mathrm{Pe}}(x, y ; \rho)=\frac{p(x) q^{\prime \prime}(y)-p^{\prime}(x) q^{\prime}(y)+p^{\prime \prime}(x) q(y)-\rho p(x) q(y)}{x-y},
$$

with

$$
p(x)=\frac{1}{2 \pi} \int_{-\infty}^{\infty} e^{-\frac{1}{4} s^{4}-\frac{\rho}{2} s^{2}+i s x} \mathrm{~d} s \quad \text { and } \quad q(y)=\frac{1}{2 \pi} \int_{\Sigma} e^{\frac{1}{4} t^{4}+\frac{\rho}{2} t^{2}+i t y} \mathrm{~d} t,
$$

where $\rho \in \mathbb{R}$. The contour $\Sigma$ consists of the four rays $\arg t=\pi / 4,3 \pi / 4,5 \pi / 4,7 \pi / 4$, where the first and the third ray are oriented from infinity to zero while the second and the last ray are oriented outwards. The functions (2.9) are called Pearcey integrals [38] and are solutions of the third order differential equations $p^{\prime \prime \prime}(x)=x p(x)+\rho p(x)$ and $q^{\prime \prime \prime}(y)=-y q(y)+\rho q(y)$, respectively.

The Pearcey kernel appears in several random models including the closing of gap in the Gaussian random matrix model with external source [13, 12, 40], non-intersecting Brownian motions at cusps [5] (see also interpretations in this paper), a combinatorial model on random partitions [37, etc. We mention that, besides the expression (2.8), the Pearcey kernel admits a double integral representation as well as a RH characterization. For the latter one, see Section 6.1.

This is our first main result. 
Theorem 2.3. Let $K^{\mathrm{tac}}$ be as defined in (2.5) and $K^{\mathrm{Pe}}$ as in (2.8). Then

$$
\lim _{a \rightarrow-\infty} \frac{1}{\sqrt{2}|a|^{1 / 4}} K^{\mathrm{tac}}\left(\frac{x}{\sqrt{2}|a|^{1 / 4}}, \frac{y}{\sqrt{2}|a|^{1 / 4}} ;-\frac{a^{2}}{2},|a|\left(1+\frac{\sigma}{2|a|^{3 / 2}}\right)\right)=K^{\mathrm{Pe}}(y, x ; \sigma),
$$

and

$$
\lim _{a \rightarrow \infty} \frac{1}{\sqrt{2} a^{1 / 4}} K^{\operatorname{tac}}\left(\frac{x}{\sqrt{2} a^{1 / 4}}, \frac{y}{\sqrt{2} a^{1 / 4}} ;-\frac{a^{2}}{2},-a\left(1+\frac{\sigma}{2 a^{3 / 2}}\right)\right)=K^{\mathrm{Pe}}(x, y ; \sigma),
$$

uniformly for $x, y$ in compact sets, where $\sigma \in \mathbb{R}$ is a parameter.

The good scaling of the parameters $s, t$ in the tacnode kernel $K^{\mathrm{tac}}$ can be derived from the phase diagram in Figure 3. Indeed, the phase diagram suggests that it is possible to retrieve the Pearcey kernel from the tacnode kernel by letting $\tau$ and $T$ move away from the multicritical point $\left(\tau_{\text {crit }}, 1\right)$ along the curve (2.3) that separates Case II from Case III. Recall the double scaling (2.4) of $\tau$ and $T$ involving $K$ and $L$. If we leave the multicritical point to the left/right along the curve, by (2.4), this amounts to setting $L=2 K^{2}(\alpha+\beta)^{4}$ and $\mp K>0$. This implies that the tacnode kernel appearing in Theorem 2.2 should take parameters $s, t$ as

$$
s=-a^{2} / 2 \quad \text { and } \quad t=-a,
$$

with $\mp a=\mp 2^{-1 / 3}(\alpha+\beta)^{2} K>0$. A further consideration noting that the Pearcey kernel is a one parameter family invokes us to take

$$
t=-a\left(1+\frac{\sigma}{2|a|^{3 / 2}}\right), \quad \sigma \in \mathbb{R}
$$

in our setting.

Theorem 2.3 will be proved in Section 6.2. In Sections 46 we will mainly focus on proving (2.10). In the end we will obtain (2.11) from (2.10) using a symmetry argument. We next state the results for the transitions in the Hermitian two-matrix model.

\section{From the critical kernel to the Pearcey kernel}

\subsection{The phase diagram}

As aforementioned, the eigenvalues of the matrix $M_{1}$ in the Hermitian two-matrix model (1.2) form a determinantal process with correlation kernel $K_{11}^{(n)}(x, y)$. In this section we will again use a phase diagram to get more insight into the nature of the critical phenomena and phase transitions involved.

By the results in [28], it follows that the mean eigenvalue density for $M_{1}$ has a limit as $n \rightarrow \infty$, i.e., there exists an absolutely continuous measure $\mu_{1}$ on $\mathbb{R}$ such that

$$
\lim _{n \rightarrow \infty} \frac{1}{n} K_{11}^{(n)}(x, x)=\frac{\mathrm{d} \mu_{1}}{\mathrm{~d} x} .
$$


For technical reasons this result was only proved under the assumption that $n \equiv 0$ mod 3. A crucial point in the analysis of [28] is that the limiting measure $\mu_{1}$ can be characterized by a vector equilibrium problem for vectors of three measures $\left(\mu_{1}, \mu_{2}, \mu_{3}\right)$, see also [32]. This equilibrium problem involves a constraint $\sigma_{2}$ acting on the second measure $\mu_{2}$, where $\sigma_{2}$ is a certain measure on the imaginary axis. Moreover, the supports of the measures $\mu_{1}, \sigma_{2}-\mu_{2}$, and $\mu_{3}$ have the following forms

$$
\begin{aligned}
\operatorname{supp}\left(\mu_{1}\right) & =\left[-\beta_{0},-\beta_{1}\right] \cup\left[\beta_{1}, \beta_{0}\right], \\
\operatorname{supp}\left(\sigma_{2}-\mu_{2}\right) & =i \mathbb{R} \backslash\left(-i \beta_{2}, i \beta_{2}\right), \\
\operatorname{supp}\left(\mu_{3}\right) & =\mathbb{R} \backslash\left(-\beta_{3}, \beta_{3}\right),
\end{aligned}
$$

for some $\beta_{0}>\beta_{1} \geq 0, \beta_{2}, \beta_{3} \geq 0$ that all depend on the parameters $\alpha \in \mathbb{R}$ and $\tau>0$. Generically, at least one of these numbers is zero, and no two consecutive ones are zero. This leads to the following classification of cases as proved in [27, 28].

Case I: $\beta_{1}=0, \beta_{2}>0$, and $\beta_{3}=0$. In this case there are no gaps in the supports of the measures $\mu_{1}$ and $\mu_{3}$ on the real line. The constraint $\sigma_{2}$ is active along an interval $\left[-i \beta_{2}, i \beta_{2}\right]$ on the imaginary axis.

Case II: $\beta_{1}>0, \beta_{2}>0$, and $\beta_{3}=0$. In Case II there is a gap in the support of $\mu_{1}$, but there is no gap in the support of $\mu_{3}$, which is again the full real line. The constraint is active along an interval along the imaginary axis.

Case III: $\beta_{1}>0, \beta_{2}=0$, and $\beta_{3}>0$. In Case III there is a gap in the supports of $\mu_{1}$ and $\mu_{3}$, but the constraint on the imaginary axis is not active.

Case IV: $\beta_{1}=0, \beta_{2}>0$, and $\beta_{3}>0$. In this case the measure $\mu_{1}$ is still supported on one interval. However there is a gap $\left(-\beta_{3}, \beta_{3}\right)$ in the support of $\mu_{3}$. As in Case I, the constraint $\sigma_{2}$ is active along an interval $\left[-i \beta_{2}, i \beta_{2}\right]$ on the imaginary axis.

The correspondences between the values of $(\alpha, \tau)$ and these four cases are illustrated in the phase diagram shown in Figure 4. The different cases are separated by two curves with equations

$$
\tau=\sqrt{\alpha+2}, \quad-2 \leq \alpha<\infty
$$

and

$$
\tau=\sqrt{-\frac{1}{\alpha}}, \quad-\infty<\alpha<0,
$$

respectively. On these curves two of the numbers $\beta_{1}, \beta_{2}$, and $\beta_{3}$ are equal to zero. For example, on the curve (3.3) that separates Case III from Case IV, we have $\beta_{1}=\beta_{2}=0$, while $\beta_{3}>0$. Finally, note the multi-critical point $(\alpha, \tau)=(-1,1)$ in the phase diagram, where $\beta_{1}=\beta_{2}=\beta_{3}=0$. All four cases come together at this point in the $\alpha \tau$-plane.

As long as we consider points $(\alpha, \tau)$ that are not on the curves, the local eigenvalue correlations are governed by the sine kernel in the bulk of the spectrum and the Airy kernel at the edge of the spectrum. Critical phenomena occur at the curves that separate the different cases. When we cross the line $\tau=\sqrt{\alpha+2}$ for $\alpha>-1$, the support of $\mu_{1}$ 


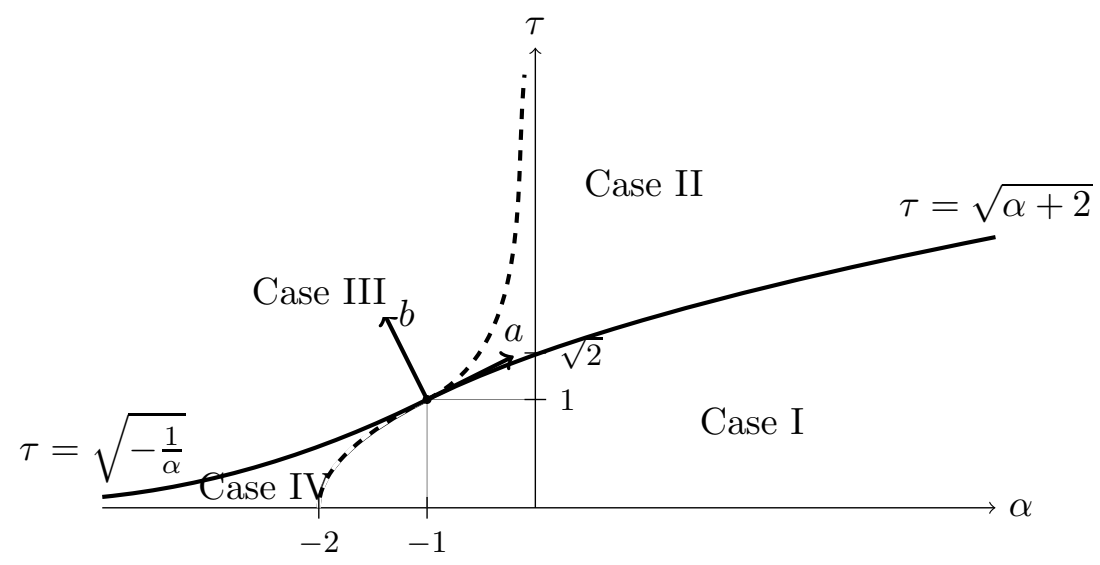

Figure 4: The phase diagram in the $\alpha \tau$-plane: the critical curves $\tau=\sqrt{\alpha+2}$ and $\tau=\sqrt{-\frac{1}{\alpha}}$ separate the four cases. The cases are distinguished by the fact whether 0 is in the support of the measures $\mu_{1}, \sigma_{2}-\mu_{2}$, and $\mu_{3}$, or not.

turns from two intervals into one interval. On this critical curve, the intervals meet at the origin and there the density vanishes quadratically. This indicates that in a double scaling limit the correlation kernel converges to the Painlevé II kernel that arises at the closing of a gap in unitary ensembles [11, 15, 16. When crossing the line $\tau=\sqrt{-1 / \alpha}$ for $\alpha<-1$, we again have a transition of two intervals merging at the origin. However, due to the fact that $\operatorname{supp}\left(\sigma_{2}-\mu_{2}\right)$ also closes simultaneously, the vanishing at the origin occurs with an exponent $1 / 3$. This indicates that the local correlations are governed by the Pearcey kernel. The other transitions, represented by the dashed lines in Figure 4 , do not concern $\mu_{1}$. They take place on the non-physical sheets of the spectral curve and, therefore, they do not influence the local correlations of the eigenvalues of $M_{1}$, which are again described by the sine and Airy kernels.

The limiting process near the multicritical point $(\alpha, \tau)=(-1,1)$, where there is a simultaneous transition in the supports of all three measures $\mu_{1}, \sigma-\mu_{2}$ and $\mu_{3}$ is the subject of the recent paper [26]. Here the limiting eigenvalue density vanishes like a square root at the interior of the support, which is similar to the situation in nonintersecting Brownian motions at a tacnode. By taking triple scaling limits such that $\alpha$ and $\tau$ are made dependent on $n$ as

$$
\left(\begin{array}{c}
\alpha \\
\tau
\end{array}\right)=\left(\begin{array}{c}
-1 \\
1
\end{array}\right)+a n^{-1 / 3}\left(\begin{array}{l}
2 \\
1
\end{array}\right)+b n^{-2 / 3}\left(\begin{array}{c}
-1 \\
2
\end{array}\right)
$$

for $a, b \in \mathbb{R}$, the large $n$ limit of the correlation kernel $K_{11}^{(n)}(x, y)$ is given by the critical kernel $K^{\mathrm{cr}}$ that we will introduce in the next section. Note that in (3.4) the vectors $\left(\begin{array}{ll}2 & 1\end{array}\right)^{T}$ and $\left(\begin{array}{ll}-1 & 2\end{array}\right)^{T}$ are respectively tangent and normal to both critical curves in the point $(\alpha, \tau)=(-1,1)$. 


\subsection{The critical kernel}

Definition 3.1. For $u, v \in \mathbb{R}$, we define the critical kernel $K^{\text {cr }}$ by

$$
K^{\mathrm{cr}}(u, v ; s, t)=\frac{1}{2 \pi i(u-v)}\left(\begin{array}{llll}
-1 & 1 & 0 & 0
\end{array}\right) M(i u ; s, t)^{-1} M(i v ; s, t)\left(\begin{array}{l}
1 \\
1 \\
0 \\
0
\end{array}\right) .
$$

The following theorem is the main result in [26].

Theorem 3.2. [26, Theorem 2.3] Let $K_{11}^{(n)}$ be the kernel describing the eigenvalues of $M_{1}$ when averaged over $M_{2}$ in the Hermitian two-matrix model (1.2) with $V$ and $W$ as in (1.3). Assume $\alpha$ and $\tau$ depend on $n$ as in (3.4). Then for $n \rightarrow \infty$ and $n \equiv 0 \bmod 6$, we have

$$
\lim _{n \rightarrow \infty} \frac{1}{n^{2 / 3}} K_{11}^{(n)}\left(\frac{u}{n^{2 / 3}}, \frac{v}{n^{2 / 3}}\right)=K^{\mathrm{cr}}\left(u, v ; \frac{1}{4}\left(a^{2}-5 b\right),-a\right),
$$

uniformly for $u, v$ in compact subsets of $\mathbb{R}$, where $K^{\mathrm{cr}}$ is defined in (3.5).

\subsection{Double scaling limit: from the critical kernel to the Pearcey kernel}

Our second main result is the following.

Theorem 3.3. Let $K^{\mathrm{cr}}$ be as defined in (3.5) and $K^{\mathrm{Pe}}$ as in (2.8). Then

$$
\lim _{a \rightarrow-\infty} \frac{1}{\sqrt{2}|a|^{1 / 4}} K^{\mathrm{cr}}\left(\frac{x}{\sqrt{2}|a|^{1 / 4}}, \frac{y}{\sqrt{2}|a|^{1 / 4}} ;-\frac{1}{2} a^{2},|a|\left(1-\frac{\sigma}{2|a|^{3 / 2}}\right)\right)=K^{\mathrm{Pe}}(x, y ; \sigma),
$$

uniformly for $x, y$ in compact sets, where $\sigma \in \mathbb{R}$ is a parameter.

The scaling of the parameters $s, t$ in the critical kernel $K^{\mathrm{cr}}$ is again motivated by the phase diagram in Figure 4. This phase diagram suggests that it is possible to retrieve the Pearcey kernel from the critical kernel by letting $t$ and $\alpha$ move away from the multicritical point along the curve $\tau=\sqrt{-1 / \alpha}, \alpha<-1$. This amounts to setting $b=3 a^{2} / 5$ and $a<0$. Hence the critical kernel in Theorem 3.2 takes parameters $s=-a^{2} / 2$ and $t=|a|$. To obtain the limit in the most general form, we perform the scaling for $t$ as

$$
t=|a|\left(1-\frac{\sigma}{2|a|^{3 / 2}}\right), \quad \sigma \in \mathbb{R} .
$$

We also note that if one lets $t$ and $\alpha$ move away from the multicritical point along the curve $\tau=\sqrt{\alpha+2}, \alpha>-1$, the critical kernel will tend to the Painlevé II kernel; see [26] for more details.

Theorem 3.3 will be proved in Section 6.3 . 


\section{Meromorphic $\lambda$-functions on a Riemann surface}

In this section, we introduce some auxiliary functions and study their properties. The aim is to construct the so-called $\lambda$-functions, of which the analytic continuation defines a meromorphic function on a Riemann surface with specified sheet structure. The $\lambda$ functions have desired behavior around each branch point, and we shall make use of them in the normalization step of our steepest descent analysis in Section 5.3.

Throughout this section, unless specified differently, we shall take the principal cut for all fractional powers.

\subsection{A four-sheeted Riemann surface and the $w$-functions}

We introduce a four-sheeted Riemann surface $\mathcal{R}$ with sheets

$$
\begin{array}{ll}
\mathcal{R}_{1}=\mathbb{C} \backslash[0, \infty), & \mathcal{R}_{3}=\mathbb{C} \backslash([-i c, i c] \cup[0, \infty)), \\
\mathcal{R}_{2}=\mathbb{C} \backslash(-\infty, 0], & \mathcal{R}_{4}=\mathbb{C} \backslash([-i c, i c] \cup(-\infty, 0]),
\end{array}
$$

where

$$
c=\frac{3 \sqrt{3}}{16} \gamma^{-3 / 2}
$$

and $\gamma>0$ is a parameter that we will specify later.

We connect the sheets $\mathcal{R}_{j}, j=1,2,3,4$, to each other in the usual crosswise manner along the cuts $[-i c, i c],[0, \infty)$, and $(-\infty, 0]$. More precisely, $\mathcal{R}_{1}$ is connected to $\mathcal{R}_{3}$ along the cut $[0, \infty), \mathcal{R}_{2}$ is connected to $\mathcal{R}_{4}$ along the cut $(-\infty, 0]$, and $\mathcal{R}_{3}$ is connected to $\mathcal{R}_{4}$ via $[-i c, i c]$. Moreover, the Riemann surface is compactified by adding two points at infinity. The first point $\infty_{1}$ is added to the first and the third sheet, while the second point $\infty_{2}$ connects the other two sheets. This Riemann surface $\mathcal{R}$ has genus zero and is shown in Figure 5 ,

We intend to find functions $\lambda_{j}$ on these sheets, such that each $\lambda_{j}$ is analytic on $\mathcal{R}_{j}$ and admits an analytic continuation across the cuts. Later we will impose extra conditions on the poles and zeros of this function. For the moment, we content ourselves constructing an elementary function $w$ that is meromorphic on $\mathcal{R}$.

Consider the algebraic equation

$$
z=\frac{w}{\left(\gamma^{3} w^{2}-1\right)^{2}}
$$

It turns out this equation defines a meromorphic function on the Riemann surface $\mathcal{R}$. This is made precise in the following lemma.

Lemma 4.1. There exist functions $w_{j}(z), j=1,2,3,4$, that solve the equation (4.2) and satisfy the following conditions.

(a) $w_{j}(z)$ is analytic on $\mathcal{R}_{j}, j=1,2,3,4$, and

$$
\begin{aligned}
& w_{1, \pm}(x)=w_{3, \mp}(x), \quad x \in(0, \infty), \\
& w_{2, \pm}(x)=w_{4, \mp}(x), \quad x \in(-\infty, 0), \\
& w_{3, \pm}(z)=w_{4, \mp}(z), \quad z \in(-i c, 0) \cup(0, i c) \text {. }
\end{aligned}
$$




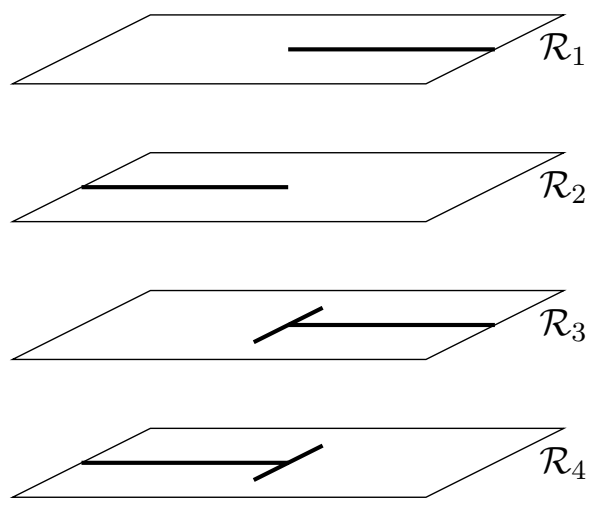

Figure 5: Riemann surface $\mathcal{R}$.

Here, we orient $(-\infty, 0)$ and $(0, \infty)$ from left to right, and $(-i c, 0) \cup(0, i c)$ from bottom to top. Hence, the function $\bigcup_{j=1}^{4} \mathcal{R}_{j} \rightarrow \mathbb{C}: \mathcal{R}_{j} \ni z \mapsto w_{j}(z)$ has an analytic continuation to a meromorphic function $w: \mathcal{R} \rightarrow \overline{\mathbb{C}}$. This function is a bijection.

(b) $w$ satisfies the symmetry properties

$$
\begin{aligned}
w_{j}(\bar{z}) & =\overline{w_{j}(z)}, & & z \in \mathcal{R}_{j}, \quad j=1,2,3,4, \\
w_{1}(-z) & =-w_{2}(z), & & z \in \mathbb{C} \backslash(-\infty, 0], \\
w_{3}(-z) & =-w_{4}(z), & & z \in \mathbb{C} \backslash((-\infty, 0] \cup[-i c, i c]) .
\end{aligned}
$$

(c) As $z \rightarrow \infty$ within $\mathbb{C} \backslash(-\infty, 0]$ we have that

$$
\begin{aligned}
w_{2}(z)=\gamma^{-3 / 2}+\frac{1}{2} \gamma^{-9 / 4} z^{-1 / 2}- & \frac{1}{64} \gamma^{-15 / 4} z^{-3 / 2} \\
& +\frac{1}{128} \gamma^{-9 / 2} z^{-2}-\frac{9}{4096} \gamma^{-21 / 4} z^{-5 / 2}+\mathcal{O}\left(z^{-7 / 2}\right), \\
w_{4}(z)=\gamma^{-3 / 2}-\frac{1}{2} \gamma^{-9 / 4} z^{-1 / 2}+ & \frac{1}{64} \gamma^{-15 / 4} z^{-3 / 2} \\
& +\frac{1}{128} \gamma^{-9 / 2} z^{-2}+\frac{9}{4096} \gamma^{-21 / 4} z^{-5 / 2}+\mathcal{O}\left(z^{-7 / 2}\right) .
\end{aligned}
$$

(d) As $z \rightarrow 0$ we have that

$$
\begin{aligned}
& w_{2}(z)=\gamma^{-2} z^{-1 / 3}+\frac{2}{3} \gamma^{-1} z^{1 / 3}-\frac{1}{3} z+\frac{28}{81} \gamma z^{5 / 3}+\mathcal{O}\left(z^{7 / 3}\right), \\
& w_{4}(z)= \begin{cases}z-2 \gamma^{3} z^{3}+9 \gamma^{6} z^{5}+\mathcal{O}\left(z^{7}\right), & \text { in } \mathbb{C} \backslash(-\infty, 0], \\
-\omega^{2} \gamma^{-2}(-z)^{-1 / 3}-\frac{2}{3} \omega \gamma^{-1}(-z)^{1 / 3}-\frac{1}{3} z-\frac{28}{81} \omega^{2} \gamma(-z)^{5 / 3}+\mathcal{O}\left(z^{7 / 3}\right), & \text { in } I I, \\
\omega^{2} \gamma^{-2} z^{-1 / 3}+\frac{2}{3} \omega \gamma^{-1} z^{1 / 3}-\frac{1}{3} z+\frac{28}{81} \omega^{2} \gamma z^{5 / 3}+\mathcal{O}\left(z^{7 / 3}\right), & \text { in III. }\end{cases}
\end{aligned}
$$

Here, I, II, III and IV stand for the four open quadrants in the complex plane and $\omega=e^{2 \pi i / 3}$. 
(e) As $z \rightarrow$ ic we have that

$$
w_{3}(z)=\frac{i}{\sqrt{3}} \gamma^{-3 / 2}+8 \cdot 3^{-7 / 4} e^{3 \pi i / 4} \gamma^{-3 / 4}(z-i c)^{1 / 2}+\mathcal{O}(z-i c),
$$

where we take the branch cut of $(z-i c)^{1 / 2}$ along $(-i \infty, i c)$ and $-\pi / 2<\arg (z-i c)<$ $\frac{3 \pi}{2}$.

(f) We have

$$
w_{3,+}((0, i c))=\left(0, \frac{i}{\sqrt{3}} \gamma^{-3 / 2}\right), \quad w_{3,-}((0, i c))=\left(i \infty, \frac{i}{\sqrt{3}} \gamma^{-3 / 2}\right) .
$$

Note that the complete behavior of $w_{j}, j=1,2,3,4$, around the origin, $\infty$, and $\pm i c$ can be obtained from the symmetry conditions in (b).

Proof. We consider the algebraic equation defined by

$$
\left(\widehat{w}^{2}+\gamma^{3}\right)^{2}=z \widehat{w}^{3},
$$

where again $\gamma$ is a positive parameter as in (4.2). For any solution $\widehat{w}(z)$ of (4.3), the function

$$
w(z)=i(\widehat{w}(i / z))^{-1}
$$

satisfies equation (4.2). Our strategy is then to construct $w_{j}$ from the solutions of (4.3) via the above transformation. Fortunately, the equation (4.3) was well-studied in [26, Section 3]. For each $z \in \mathbb{C}$, it was shown that the equation (4.3) admits four solutions, denoted by $\widehat{w}_{j}(z), j=1,2,3,4$, such that

$$
\left|\widehat{w}_{1}(z)\right| \geq\left|\widehat{w}_{2}(z)\right| \geq\left|\widehat{w}_{3}(z)\right| \geq\left|\widehat{w}_{4}(z)\right| .
$$

These functions can be interpreted as a meromorphic function on another four-sheeted Riemann surface $\hat{\mathcal{R}}$ with sheets

$$
\begin{array}{ll}
\hat{\mathcal{R}}_{1}=\mathbb{C} \backslash[-\hat{c}, \hat{c}], & \hat{\mathcal{R}}_{3}=\mathbb{C} \backslash(\mathbb{R} \cup i \mathbb{R}), \\
\hat{\mathcal{R}}_{2}=\mathbb{C} \backslash([-\hat{c}, \hat{c}] \cup i \mathbb{R}), & \hat{\mathcal{R}}_{4}=\mathbb{C} \backslash \mathbb{R},
\end{array}
$$

where

$$
\hat{c}=1 / c=\frac{16}{3 \sqrt{3}} \gamma^{3 / 2} .
$$

These four sheets are connected in the usual crosswise manner and the Riemann surface is compactified by adding two points at infinity: one on the first sheet, and another to the three remaining sheets. Each $\widehat{w}_{j}(z)$ is analytic on $\hat{\mathcal{R}}_{j}$ and the function $\bigcup_{j=1}^{4} \hat{\mathcal{R}}_{j} \rightarrow$ $\mathbb{C}: \hat{\mathcal{R}}_{j} \ni z \mapsto \widehat{w}_{j}(z)$ has an analytic continuation to a meromorphic function $\widehat{w}: \hat{\mathcal{R}} \rightarrow \overline{\mathbb{C}}$. This fact invokes us to define the following functions $w_{j}(z)$ :

$$
\begin{aligned}
& w_{1}(z)=\left\{\begin{array}{ll}
i\left(\widehat{w}_{3}(i / z)\right)^{-1}, & \text { in } I \cup I V, \\
i\left(\widehat{w}_{4}(i / z)\right)^{-1}, & \text { in } I I \cup I I I,
\end{array} \quad w_{2}(z)= \begin{cases}i\left(\widehat{w}_{4}(i / z)\right)^{-1}, & \text { in } I \cup I V, \\
i\left(\widehat{w}_{3}(i / z)\right)^{-1}, & \text { in } I I \cup I I I,\end{cases} \right. \\
& w_{3}(z)=\left\{\begin{array}{ll}
i\left(\widehat{w}_{2}(i / z)\right)^{-1}, & \text { in } I \cup I V, \\
i\left(\widehat{w}_{1}(i / z)\right)^{-1}, & \text { in } I I \cup I I I,
\end{array} \quad w_{4}(z)= \begin{cases}i\left(\widehat{w}_{1}(i / z)\right)^{-1}, & \text { in } I \cup I V, \\
i\left(\widehat{w}_{2}(i / z)\right)^{-1}, & \text { in } I I \cup I I I .\end{cases} \right.
\end{aligned}
$$


Then, the lemma follows from [26, Lemmas 3.1-3.3] and straightforward computations.

\subsection{The $\lambda$-functions}

With the functions $w_{j}(z)$ in Lemma 4.1, we define the $\lambda$-functions as

$$
\lambda_{j}(z)=z^{2}\left(C_{1} w_{j}(z)^{4}+C_{2} w_{j}(z)^{2}+C_{3}+C_{4} w_{j}(z)^{-2}\right), \quad z \in \mathcal{R}_{j}, \quad j=1,2,3,4,
$$

where

$$
\begin{array}{ll}
C_{1}=-\frac{3}{2} \gamma^{33 / 4}+\frac{3}{32} \gamma^{27 / 4}, & C_{2}=\frac{7}{2} \gamma^{21 / 4}+\frac{17}{32} \gamma^{15 / 4}, \\
C_{3}=-\frac{5}{2} \gamma^{9 / 4}-\frac{65}{96} \gamma^{3 / 4}, & C_{4}=\frac{1}{2} \gamma^{-3 / 4}+\frac{5}{96} \gamma^{-9 / 4},
\end{array}
$$

are constants depending on $\gamma$. We now fix $\gamma$ as

$$
\gamma=4^{-4 / 3}\left(-2 \mp \frac{\sigma}{|a|^{3 / 2}}+\sqrt{5+4\left(1 \pm \frac{\sigma}{2|a|^{3 / 2}}\right)^{2}}\right)^{4 / 3}>0,
$$

with $a<0$ and $\sigma \in \mathbb{R}$. In (4.5) and the rest of the paper we choose the top sign when we are in the Brownian paths setting and the bottom sign when dealing with the two-matrix model. It is readily seen that $\gamma$ satisfies

$$
\frac{5-16 \gamma^{3 / 2}}{16 \gamma^{3 / 4}}=1 \pm \frac{\sigma}{2|a|^{3 / 2}}
$$

and

$$
\gamma=4^{-4 / 3}\left(1 \mp \frac{4 \sigma}{9|a|^{3 / 2}}+\mathcal{O}\left(a^{-3}\right)\right)
$$

as $a \rightarrow-\infty$ with $\sigma$ fixed.

Since $\gamma$ depends on the parameters $a$ and $\sigma$, the functions and constants $w_{j}, \lambda_{j}, c, C_{j}$ as well as some functions and constants to be introduced later, depend on $a$ and $\sigma$ through $\gamma$, although this is not indicated explicitly. Recall that we are interested in the behavior of these notions for large negative $a$. We will add ${ }^{*}$ to the notation to denote these functions or constants in the limit $a \rightarrow-\infty$ with $\sigma$ fixed.

The properties of the $\lambda$-functions are listed in the following lemma.

Lemma 4.2. The functions $\lambda_{j}(z), j=1,2,3,4$, defined by (4.4) have the following properties.

(a) $\lambda_{j}(z)$ is analytic on $\mathcal{R}_{j}, j=1,2,3,4$, and

$$
\begin{aligned}
& \lambda_{1, \pm}(x)=\lambda_{3, \mp}(x), \quad x \in(0, \infty), \\
& \lambda_{2, \pm}(x)=\lambda_{4, \mp}(x), \quad x \in(-\infty, 0), \\
& \lambda_{3, \pm}(z)=\lambda_{4, \mp}(z), \quad z \in(-i c, 0) \cup(0, i c) .
\end{aligned}
$$

Hence the function $\bigcup_{j=1}^{4} \mathcal{R}_{j} \rightarrow \mathbb{C}: \mathcal{R}_{j} \ni z \mapsto \lambda_{j}(z)$ has an analytic continuation to a meromorphic function on the Riemann surface $\mathcal{R}$. 
(b) We have the following symmetry properties

$$
\begin{aligned}
\lambda_{j}(\bar{z}) & =\overline{\lambda_{j}(z)} & & z \in \mathcal{R}_{j}, \quad j=1,2,3,4, \\
\lambda_{1}(-z) & =\lambda_{2}(z), & & z \in \mathbb{C} \backslash(-\infty, 0], \\
\lambda_{3}(-z) & =\lambda_{4}(z), & & z \in \mathbb{C} \backslash((-\infty, 0] \cup[-i c, i c]) .
\end{aligned}
$$

(c) As $z \rightarrow \infty$ within $\mathbb{C} \backslash[0, \infty)$, we have

$$
\begin{aligned}
& \lambda_{2}(z)=\frac{2}{3} z^{3 / 2}+\left(1 \pm \frac{\sigma}{2|a|^{3 / 2}}\right) z-z^{1 / 2}+\ell-2 D z^{-1 / 2}+\mathcal{O}\left(z^{-1}\right), \\
& \lambda_{4}(z)=-\frac{2}{3} z^{3 / 2}+\left(1 \pm \frac{\sigma}{2|a|^{3 / 2}}\right) z+z^{1 / 2}+\ell+2 D z^{-1 / 2}+\mathcal{O}\left(z^{-1}\right),
\end{aligned}
$$

where $\ell$ and $D$ are complex constants depending on $\gamma$.

(d) For $z$ in a neighborhood of the origin we have

$$
\begin{array}{ll}
\lambda_{2}(z)=G(z) z^{2 / 3}+H(z) z^{4 / 3}+K(z) z^{2}, & \text { in } \mathbb{C} \backslash(-\infty, 0], \\
\lambda_{4}(z)= \begin{cases}L(z), & \text { in } I \cup I V, \\
G(z) \omega z^{2 / 3}+H(z) \omega^{2} z^{4 / 3}+K(z) z^{2}, & \text { in } I I, \\
G(z) \omega^{2} z^{2 / 3}+H(z) \omega z^{4 / 3}+K(z) z^{2}, & \text { in } I I I,\end{cases}
\end{array}
$$

where $G(z), H(z), K(z)$, and $L(z)$ are even analytic functions in a neighborhood of the origin with

$$
\begin{aligned}
G(0) & =\frac{3}{32 \gamma^{5 / 4}}\left(1-16 \gamma^{3 / 2}\right), & G^{*}(0) & =0, \\
H(0) & =\frac{1}{32 \gamma^{1 / 4}}\left(25-16 \gamma^{3 / 2}\right), & H^{*}(0) & =3 \cdot 4^{-2 / 3}, \\
K(0) & =\frac{1}{96} \gamma^{3 / 4}\left(15+16 \gamma^{3 / 2}\right), & K^{*}(0) & =\frac{1}{24}, \\
L(0) & =\frac{1}{96 \gamma^{9 / 4}}\left(5+48 \gamma^{3 / 2}\right), & L^{*}(0) & =\frac{16}{3} .
\end{aligned}
$$

(e) As $z \rightarrow$ ic we have that

$$
\lambda_{3}(z)=\widetilde{G}(z)+\widetilde{H}(z)(z-i c)^{3 / 2},
$$

where we take the branch cut of $(z-i c)^{1 / 2}$ along $(-i \infty, i c)$ and $-\pi / 2<\arg (z-i c)<$ $\frac{3 \pi}{2}$. Here $\widetilde{G}$ and $\widetilde{H}$ are analytic functions in a neighborhood of ic satisfying

$$
\begin{array}{ll}
\widetilde{G}(i c)=\frac{9}{256 \gamma^{9 / 4}}\left(3+16 \gamma^{3 / 2}\right), & \widetilde{G}^{*}\left(i c^{*}\right)=9, \\
\widetilde{H}(i c)=-\frac{3^{1 / 4}}{27}\left(5+16 \gamma^{3 / 2}\right) e^{3 \pi i / 4}, & \widetilde{H}^{*}\left(i c^{*}\right)=-\frac{2}{3^{7 / 4}} e^{3 \pi i / 4} .
\end{array}
$$


Note that the complete behavior of $\lambda_{j}, j=1,2,3,4$, around the origin, $\infty$, and $\pm i c$ can be obtained from the symmetry conditions in (b).

Proof. The proof is straightforward using (4.4) and Lemma 4.1.

As $a \rightarrow-\infty$, the functions $\lambda_{j}$ converge to $\lambda_{j}^{*}$, i.e., the $\lambda$-functions associated with the parameter $\gamma^{*}=4^{-4 / 3}$. The convergence will be uniform in compact subsets of $\mathbb{C}$. For later use, we need the following estimate of the convergence rate.

Lemma 4.3. There exists a constant $\varrho>0$ such that, for every large $a$, we have

$$
\left|\lambda_{j}(z)-\lambda_{j}^{*}(z)\right| \leq \varrho|a|^{-3 / 2} \max \left(1,|z|^{3 / 2}\right), \quad z \in \mathbb{C} \backslash D(0, \delta),
$$

for $j=1,2,3,4$, where $D(0, \delta)$ is an open disk centered at the origin with small radius $\delta>0$.

Proof. Due to (4.7), we have

$$
\gamma-\gamma^{*}=\mathcal{O}\left(|a|^{-3 / 2}\right), \quad \text { as } a \rightarrow-\infty .
$$

Together with (4.2) and (4.4), this implies

$$
\left|\lambda_{j}(z)-\lambda_{j}^{*}(z)\right|=\mathcal{O}\left(|a|^{-3 / 2}\right), \quad \text { as } a \rightarrow-\infty,
$$

where the constant is uniform for $z$ in compact subsets of $\mathbb{C}$. Combining this with the behavior of the $\lambda$-functions at infinity, as given in Lemma 4.2 (c), we obtain the lemma.

\section{$5 \quad$ Steepest descent analysis for $M$}

In this section we perform the steepest descent analysis of the tacnode $\mathrm{RH}$ problem 1.1. As discussed in Sections 2.3 and 3.3, we want to study the solution $M$ of this RH problem for the following values of parameters

$$
r_{1}=r_{2}=1
$$

and

$$
s_{1}=s_{2}=-\frac{a^{2}}{2}, \quad t=|a|\left(1 \pm \frac{\sigma}{2|a|^{3 / 2}}\right),
$$

as $a$ tends to $-\infty$. For the Brownian paths setting it is more convenient to choose the plus sign in the formula for $t$, whereas the minus sign is more appropriate in the twomatrix model. The analysis consists of a series of explicit and invertible transformations

$$
M \mapsto M^{(1)} \mapsto M^{(2)} \mapsto M^{(3)} \mapsto M^{(4)} \mapsto M^{(5)}
$$

of the $\mathrm{RH}$ problem. In Section 6 we will use these transformations to prove Theorems 2.3 and 3.3 . Since we are interested in the case $a \rightarrow-\infty$, we may assume that $a<0$. 


\subsection{First transformation: $M \mapsto M^{(1)}$}

This transformation is a rescaling of the $\mathrm{RH}$ problem for $M$. Define

$$
M^{(1)}(z ; a)=\operatorname{diag}\left(|a|^{1 / 2},|a|^{1 / 2},|a|^{-1 / 2},|a|^{-1 / 2}\right) M\left(a^{2} z ;-\frac{a^{2}}{2},|a|\left(1 \pm \frac{\sigma}{2|a|^{3 / 2}}\right)\right) .
$$

Then $M^{(1)}$ satisfies the following RH problem.

Lemma 5.1. The function $M^{(1)}$ defined in (5.2) has the following properties

(1) $M^{(1)}(z)$ is analytic for $z \in \mathbb{C} \backslash \Sigma_{M}$, where $\Sigma_{M}$ is shown in Figure Q.

(2) $M^{(1)}$ has the same jump matrices on $\Sigma_{M}$ as $M$; see Figure Q

(3) As $z \rightarrow \infty$ with $z \in \mathbb{C} \backslash \Sigma_{M}$, we have

$$
\begin{aligned}
& M^{(1)}(z)=\left(I+\mathcal{O}\left(z^{-1}\right)\right) B(z) A \\
& \quad \times \operatorname{diag}\left(e^{|a|^{3}(-\widetilde{\psi}(-z)+\widetilde{t} z)}, e^{|a|^{3}(-\widetilde{\psi}(z)-\widetilde{t} z)}, e^{|a|^{3}(\widetilde{\psi}(-z)+\widetilde{t} z)}, e^{|a|^{3}(\widetilde{\psi}(z)-\widetilde{t} z)}\right),
\end{aligned}
$$

where $A$ and $B(z)$ are given in (1.7) $-(1.8)$, and

$$
\widetilde{\psi}(z)=\frac{2}{3} z^{3 / 2}-z^{1 / 2}, \quad \widetilde{t}=1 \pm \frac{\sigma}{2|a|^{3 / 2}} .
$$

(4) $M^{(1)}$ is bounded near the origin.

Proof. This is immediate from RH problem 1.1 and (5.2).

\subsection{Second and third transformations: $M^{(1)} \mapsto M^{(2)} \mapsto M^{(3)}$}

It is the aim of the second and the third transformation to eliminate the jump matrices on $\Gamma_{j}, j=2,3,7,8$, see Figure 2. For convenience, we fix the angles $\varphi_{1}=\pi / 4$ and $\varphi_{2}=\pi / 3$. In a first transformation $M^{(1)} \mapsto M^{(2)}$ we erase the lower right block of the jump matrices on these rays by moving them to the shifted rays emanating from $i c$ (for $\Gamma_{2}$ and $\Gamma_{3}$ ) and $-i c$ (for $\Gamma_{7}$ and $\Gamma_{8}$ ). Thus, we introduce the new rays

$$
\widetilde{\Gamma}_{2,3}=\Gamma_{2,3}+i c, \quad \widetilde{\Gamma}_{7,8}=\Gamma_{7,8}-i c
$$

and define $M^{(2)}$ as follows. For $k=2,3,7,8$ it is given by

$$
M^{(2)}(z)= \begin{cases}M^{(1)}(z)\left(I-E_{3,4}\right), & k=2,8, \\ M^{(1)}(z)\left(I-E_{4,3}\right), & k=3,7,\end{cases}
$$

in the region bounded by $\Gamma_{k}, \widetilde{\Gamma}_{k}$, and $i \mathbb{R}$. Here $E_{i, j}$ denotes the $4 \times 4$ elementary matrix with entry 1 at the $(i, j)$ th position and all other entries equal to zero. In the remaining regions we simply set $M^{(2)}=M^{(1)}$. 
In the next transformation $M^{(2)} \mapsto M^{(3)}$ we eliminate the remaining upper left block of the jumps on $\Gamma_{j}, j=2,3,7,8$, by, respectively, moving it to the rays $\Gamma_{j}, j=1,4,6,9$. This completely eliminates the jump matrices on $\Gamma_{j}, j=2,3,7,8$. Thus, for $k=1,3,6,8$, we define

$$
M^{(3)}(z)= \begin{cases}M^{(2)}(z)\left(I-E_{2,1}\right), & k=1,8, \\ M^{(2)}(z)\left(I-E_{1,2}\right), & k=3,6,\end{cases}
$$

for $z$ in the sector bounded by $\Gamma_{k}$ and $\Gamma_{k+1}$. In the other regions we put $M^{(3)}=M^{(2)}$.

A straightforward check then yields that $M^{(3)}$ has jumps on the contour

$$
\Sigma_{M^{(3)}}:=\mathbb{R} \cup e^{\pi i / 4} \mathbb{R} \cup e^{-i \pi / 4} \mathbb{R} \cup \widetilde{\Gamma}_{2} \cup \widetilde{\Gamma}_{3} \cup \widetilde{\Gamma}_{7} \cup \widetilde{\Gamma}_{8} \cup[-i c, i c],
$$

as illustrated in Figure 6, and satisfies the following RH problem.

Lemma 5.2. The function $M^{(3)}$ as defined in (5.6) has the properties

(1) $M^{(3)}(z)$ is analytic for $z \in \mathbb{C} \backslash \Sigma_{M^{(3)}}$.

(2) $M_{+}^{(3)}(z)=M_{-}^{(3)}(z) J_{M^{(3)}}(z)$, for $z \in \Sigma_{M^{(3)}}$, where the jump matrices $J_{M^{(3)}}(z)$ are shown in Figure [6. Note that we reversed the orientation of some rays.

(3) As $z \rightarrow \infty, M^{(3)}$ has the same asymptotics as $M^{(1)}$; see (5.3).

(4) $M^{(3)}$ is bounded near the origin and near $\pm i c$.

\subsection{Fourth transformation: $M^{(3)} \mapsto M^{(4)}$}

In the fourth transformation we normalize the behavior around infinity using the $\lambda$ functions introduced in Section 4.2, We define

$$
\begin{aligned}
M^{(4)}(z)=e^{-|a|^{3} \ell}\left(I-2 i D|a|^{3} E_{3,1}\right. & \left.+2 i D|a|^{3} E_{4,2}\right) M^{(3)}(z) \\
& \times \operatorname{diag}\left(e^{|a|^{3} \lambda_{1}(z)}, e^{|a|^{3} \lambda_{2}(z)}, e^{|a|^{3} \lambda_{3}(z)}, e^{|a|^{3} \lambda_{4}(z)}\right),
\end{aligned}
$$

where $\ell$ and $D$ are the constants in property (c) of Lemma 4.2.

Then $M^{(4)}$ satisfies the following RH problem with $\Sigma_{M^{(4)}}:=\Sigma_{M^{(3)}}$.

Lemma 5.3. The function $M^{(4)}$, as defined in (5.8), has the following properties

(1) $M^{(4)}(z)$ is analytic for $z \in \mathbb{C} \backslash \Sigma_{M^{(4)}}$.

(2) For $z \in \Sigma_{M^{(4)}}$, we have $M_{+}^{(4)}(z)=M_{-}^{(4)}(z) J_{M^{(4)}}(z)$, where

$$
\begin{aligned}
J_{M^{(4)}}(z)= & \operatorname{diag}\left(e^{-|a|^{3} \lambda_{1,-}(z)}, e^{-|a|^{3} \lambda_{2,-}(z)}, e^{-|a|^{3} \lambda_{3,-}(z)}, e^{-|a|^{3} \lambda_{4,-}(z)}\right) \\
& \times J_{M^{(3)}}(z) \operatorname{diag}\left(e^{|a|^{3} \lambda_{1,+}(z)}, e^{|a|^{3} \lambda_{2,+}(z)}, e^{|a|^{3} \lambda_{3,+}(z)}, e^{|a|^{3} \lambda_{4,+}(z)}\right) .
\end{aligned}
$$




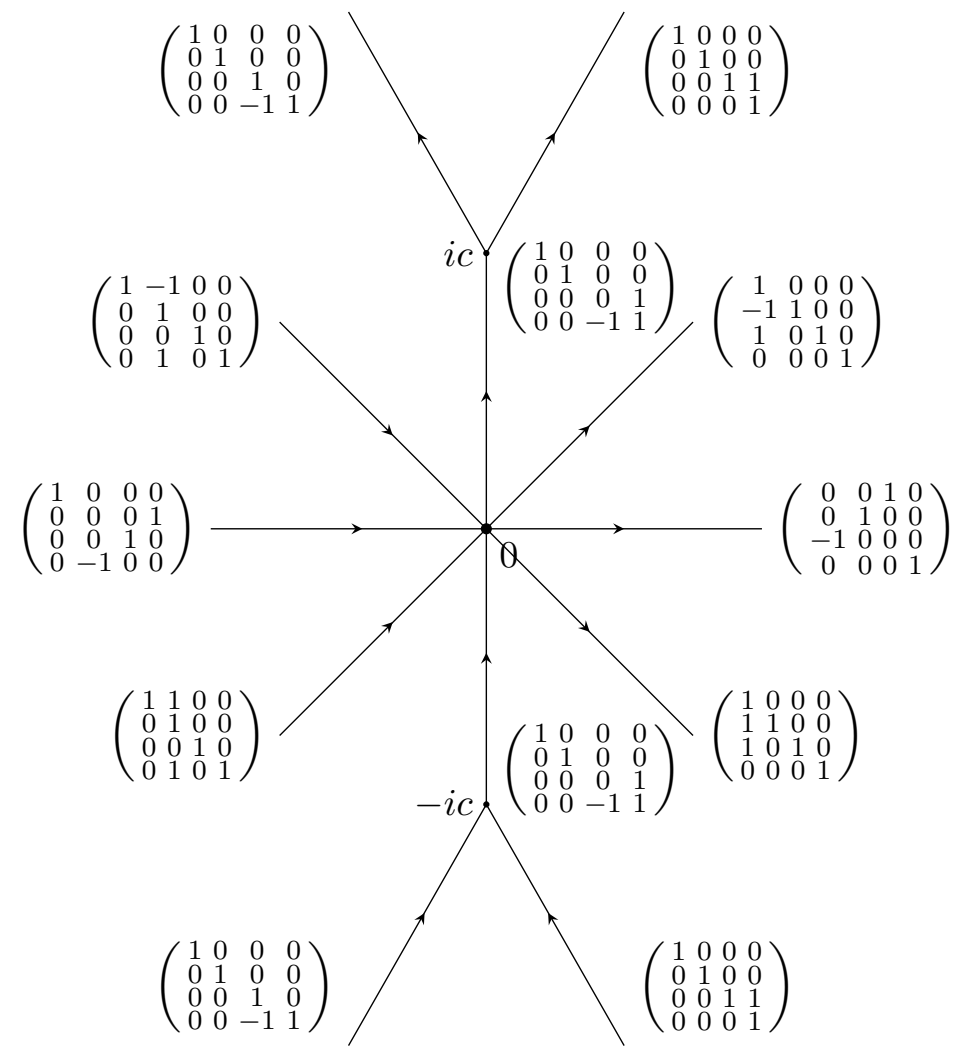

Figure 6: The jump contour $\Sigma_{M^{(3)}}$ and jump matrices for $M^{(3)}(z)$.

(3) As $z \rightarrow \infty$ we have

$$
M^{(4)}(z)=\left(I+\mathcal{O}\left(z^{-1}\right)\right) B(z) A
$$

(4) $M^{(4)}$ is bounded near the origin and near $\pm i c$.

The jump matrices $J_{M^{(4)}}(z)$ are explicitly given by

$$
\begin{gathered}
J_{M^{(4)}}(z)=\left(\begin{array}{cccc}
1 & 0 & 0 & 0 \\
0 & 0 & 0 & 1 \\
0 & 0 & 1 & 0 \\
0 & -1 & 0 & 0
\end{array}\right), \quad z \in(-\infty, 0), \quad J_{M^{(4)}}(z)=\left(\begin{array}{cccc}
0 & 0 & 1 & 0 \\
0 & 1 & 0 & 0 \\
-1 & 0 & 0 & 0 \\
0 & 0 & 0 & 1
\end{array}\right), \quad z \in(0, \infty), \\
J_{M^{(4)}}(z)=\left(\begin{array}{cccc}
1 & 0 & 0 & 0 \\
0 & 1 & 0 & 0 \\
0 & 0 & 0 & 1 \\
0 & 0 & -1 & e^{|a|^{3}\left(\lambda_{4,+}(z)-\lambda_{4,-}(z)\right)}
\end{array}\right),
\end{gathered}
$$


and

$$
\begin{array}{lll}
J_{M^{(4)}}(z)=I-e^{|a|^{3}\left(\lambda_{1}(z)-\lambda_{2}(z)\right)} E_{2,1}+e^{|a|^{3}\left(\lambda_{1}(z)-\lambda_{3}(z)\right)} E_{3,1}, & & z \in \Gamma_{1}, \\
J_{M^{(4)}}(z)=I-e^{|a|^{3}\left(\lambda_{2}(z)-\lambda_{1}(z)\right)} E_{1,2}+e^{|a|^{3}\left(\lambda_{2}(z)-\lambda_{4}(z)\right)} E_{4,2}, & & z \in \Gamma_{4}, \\
J_{M^{(4)}}(z)=I+e^{|a|^{3}\left(\lambda_{2}(z)-\lambda_{1}(z)\right)} E_{1,2}+e^{|a|^{3}\left(\lambda_{2}(z)-\lambda_{4}(z)\right)} E_{4,2}, & & z \in \Gamma_{6}, \\
J_{M^{(4)}}(z)=I+e^{|a|^{3}\left(\lambda_{1}(z)-\lambda_{2}(z)\right)} E_{2,1}+e^{|a|^{3}\left(\lambda_{1}(z)-\lambda_{3}(z)\right)} E_{3,1}, & & z \in \Gamma_{9}, \\
J_{M^{(4)}}(z)=I+e^{|a|^{3}\left(\lambda_{4}(z)-\lambda_{3}(z)\right)} E_{3,4}, & & z \in \widetilde{\Gamma}_{2} \cup \widetilde{\Gamma}_{8}, \\
J_{M^{(4)}}(z)=I-e^{|a|^{3}\left(\lambda_{3}(z)-\lambda_{4}(z)\right)} E_{4,3}, & & z \in \widetilde{\Gamma}_{3} \cup \widetilde{\Gamma}_{7} .
\end{array}
$$

Proof. The explicit formulas of $J_{M^{(4)}}$ follow from (5.9) and condition (a) in Lemma 4.2.

To establish the large $z$ behavior of $M^{(4)}$ shown in item (3), we first observe from condition (c) in Lemma 4.2 and (5.4) that

$$
\begin{aligned}
e^{|a|^{3}\left(\lambda_{1}(z)-\widetilde{\psi}(-z)+\tilde{t} z-\ell\right)} & =1-2 D|a|^{3}(-z)^{-1 / 2}+\mathcal{O}\left(z^{-1}\right), \\
e^{|a|^{3}\left(\lambda_{2}(z)-\widetilde{\psi}(z)-\tilde{t} z-\ell\right)} & =1-2 D|a|^{3} z^{-1 / 2}+\mathcal{O}\left(z^{-1}\right), \\
e^{|a|^{3}\left(\lambda_{3}(z)+\widetilde{\psi}(-z)+\tilde{t} z-\ell\right)} & =1+2 D|a|^{3}(-z)^{-1 / 2}+\mathcal{O}\left(z^{-1}\right), \\
e^{|a|^{3}\left(\lambda_{4}(z)+\widetilde{\psi}(z)-\tilde{t} z-\ell\right)} & =1+2 D|a|^{3} z^{-1 / 2}+\mathcal{O}\left(z^{-1}\right),
\end{aligned}
$$

as $z \rightarrow \infty$. Hence, from the asymptotic behavior of $M^{(3)}$ stated in item (3) of Lemma 5.2 , it is readily seen that

$$
\begin{aligned}
& e^{-|a|^{3} \ell} M^{(4)}(z) \operatorname{diag}\left(e^{|a|^{3} \lambda_{1}(z)}, e^{|a|^{3} \lambda_{2}(z)}, e^{|a|^{3} \lambda_{3}(z)}, e^{|a|^{3} \lambda_{4}(z)}\right)=\left(I+\mathcal{O}\left(z^{-1}\right)\right) B(z) A \\
& \quad \times\left(I+2 D|a|^{3} \operatorname{diag}\left(-(-z)^{-1 / 2},-z^{-1 / 2},(-z)^{-1 / 2}, z^{-1 / 2}\right)+\operatorname{diag}\left(\mathcal{O}\left(z^{-1}\right)\right)\right),
\end{aligned}
$$

as $z \rightarrow \infty$, where we recall $A$ and $B(z)$ are defined in (1.7)-(1.8). Note that

$$
\begin{array}{r}
B(z) A\left(I+2 D|a|^{3} \operatorname{diag}\left(-(-z)^{-1 / 2},-z^{-1 / 2},(-z)^{-1 / 2}, z^{-1 / 2}\right)+\operatorname{diag}\left(\mathcal{O}\left(z^{-1}\right)\right)\right) \\
=\left(I+2 i D|a|^{3} E_{3,1}-2 i D|a|^{3} E_{4,2}+\mathcal{O}\left(z^{-1}\right)\right) B(z) A .
\end{array}
$$

This, together with (5.8) and (5.11), implies (5.10).

\subsection{Estimate of $J_{M^{(4)}}$ on $\Sigma_{M^{(4)}}$}

Let us have a closer look at the jump matrices $J_{M^{(4)}}$ defined in (5.9). The jump matrix is constant on $(-\infty, 0)$ and on $(0, \infty)$. On $(-i c, 0) \cup(0, i c)$ there is a nonconstant $(4,4)$ entry. However, this entry is exponentially small as $a \rightarrow-\infty$. Moreover, the decay is uniform for $z$ bounded away from the branch points $\pm i c^{*}$, where $c^{*}=\lim _{a \rightarrow-\infty} c=3 \sqrt{3}$; see (4.1) and (4.7). Also on the other parts of $\Sigma_{M^{(4)}}$ the nonzero off-diagonal entries of the jump matrices turn out to be exponentially small for large $|a|$. Again the decay is uniform if we exclude small disks around 0 and $\pm i c^{*}$. 
The preceding statements can be seen as a corollary of the following estimates. We denote with $D\left(z_{0}, \delta\right)$ the fixed open disk centered at $z_{0}$ with small radius $\delta>0$ and let $\partial D\left(z_{0}, \delta\right)$ stand for its boundary. Recall also that we fixed the angles $\varphi_{1}=\pi / 4$ and $\varphi_{2}=\pi / 3$.

Lemma 5.4 (Estimates for $\lambda_{j}$ on $\left.\Sigma_{M^{(4)}}\right)$.

(a) There exist constants $c_{1}, c_{2}>0$ such that

$$
\begin{array}{ll}
\operatorname{Re}\left(\lambda_{1}(z)-\lambda_{2}(z)\right) \leq-c_{1}|z|^{2 / 3}, & \text { for } z \in\left(\Gamma_{1} \cup \Gamma_{9}\right) \backslash D(0, \delta), \\
\operatorname{Re}\left(\lambda_{1}(z)-\lambda_{3}(z)\right) \leq-c_{2}|z|^{2 / 3}, & \text { for } z \in\left(\Gamma_{1} \cup \Gamma_{9}\right) \backslash D(0, \delta), \\
\operatorname{Re}\left(\lambda_{2}(z)-\lambda_{1}(z)\right) \leq-c_{1}|z|^{2 / 3}, & \text { for } z \in\left(\Gamma_{4} \cup \Gamma_{6}\right) \backslash D(0, \delta), \\
\operatorname{Re}\left(\lambda_{2}(z)-\lambda_{4}(z)\right) \leq-c_{2}|z|^{2 / 3}, & \text { for } z \in\left(\Gamma_{4} \cup \Gamma_{6}\right) \backslash D(0, \delta),
\end{array}
$$

for $|a|$ large enough.

(b) There exists a constant $c_{3}>0$ such that

$$
\begin{array}{ll}
\operatorname{Re}\left(\lambda_{4}(z)-\lambda_{3}(z)\right) \leq-c_{3}|z|^{3 / 2}, & \text { for } z \in \widetilde{\Gamma}_{2} \cup \widetilde{\Gamma}_{8} \backslash D\left(i c^{*}, \delta\right) \\
\operatorname{Re}\left(\lambda_{3}(z)-\lambda_{4}(z)\right) \leq-c_{3}|z|^{3 / 2}, & \text { for } z \in \widetilde{\Gamma}_{3} \cup \widetilde{\Gamma}_{7} \backslash D\left(i c^{*}, \delta\right),
\end{array}
$$

if $|a|$ is large enough.

(c) There exists a constant $c_{4}>0$ such that

$$
\operatorname{Re}\left(\lambda_{4,+}(z)-\lambda_{4,-}(z)\right) \leq-c_{4},
$$

for $z \in\left[i\left(-c^{*}+\delta\right), 0\right) \cup\left(0, i\left(c^{*}-\delta\right)\right]$ if $|a|$ is sufficiently large.

Proof. Let us start with claim (a). It is sufficient to prove the first two estimates on $\Gamma_{1}$, since the remaining estimates follow from these ones by the symmetry conditions (b) in Lemma 4.2. Moreover, due to Lemma 4.3 and the triangle inequality it suffices to prove these estimates for the critical $\lambda$-functions $\lambda_{j}^{*}, j=1,2,3,4$.

Note that $\Gamma_{1}=[0, \exp i \pi / 4 \infty)$. For large values of $z \in \Gamma_{1}$, the estimates follow from the asymptotics of $\lambda_{j}^{*}$, which can be obtained by taking $a \rightarrow-\infty$ in Lemma 4.2(c). Using an extra argument one can extend the estimates to $\Gamma_{1} \backslash D(0, \delta)$. This is illustrated in Figure 7(a). This plots shows the values of $\operatorname{Re} \lambda_{j}^{*}(z), j=1,2,3,4$, for $z \in \Gamma_{1}$. It is clearly seen that $\operatorname{Re}\left(\lambda_{1}^{*}(z)-\lambda_{j}^{*}(z)\right)<0, j=2,3$, for $z \in \Gamma_{1} \backslash D(0, \delta)$. This completes the proof of (a).

The proof of (b) is analogous and is based on the plot in Figure 7 (b). We recall that $\widetilde{\Gamma}_{2}=i c^{*}+[0, \exp i \pi / 3 \infty)$. Also the proof of (c) is analogous and supported by Figure 8.

Lemma 5.4 has the following immediate corollary.

Corollary 5.5 (Estimates of $J_{M^{(4)}}$ on $\left.\Sigma_{M^{(4)}}\right)$. 


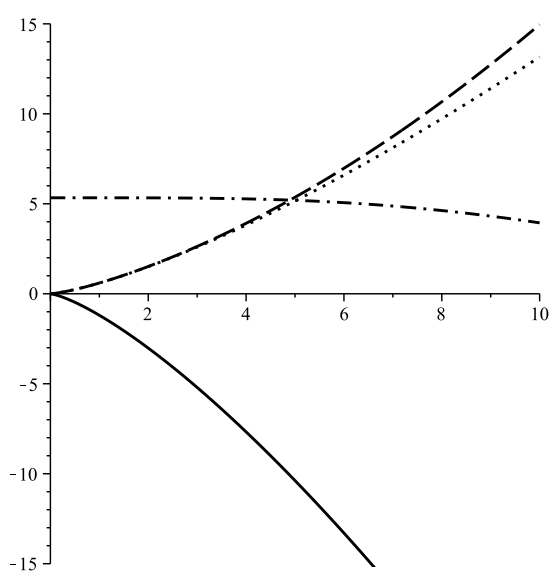

(a)

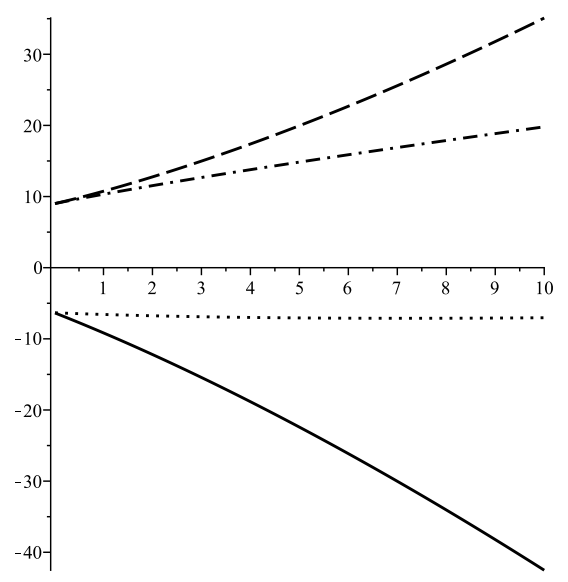

(b)

Figure 7: Plots of $\operatorname{Re} \lambda_{j}^{*}(z)$ for $j=1$ (solid), $j=2$ (dotted), $j=3$ (dashed), and $j=4$ (dashdotted). In picture (a), $z \in \Gamma_{1}$, i.e. $z=e^{\pi i / 4} x$ with $x$ on the horizontal axis. In picture (b), $z \in \widetilde{\Gamma}_{2}$, i.e. $z=i c^{*}+e^{\pi i / 3} x$ with $x$ on the horizontal axis.

(a) There is a constant $c_{1}>0$ such that

$$
J_{M^{(4)}}(z)=I+O\left(e^{-c_{1}|a|^{3}|z|^{2 / 3}}\right) \quad \text { as } a \rightarrow-\infty,
$$

uniformly for $z \in \Sigma_{M^{(4)}} \backslash\left(\mathbb{R} \cup\left[-i c^{*}, i c^{*}\right] \cup D(0, \delta) \cup D\left( \pm i c^{*}, \delta\right)\right)$.

(b) There is a constant $c_{2}>0$ such that

$$
J_{M^{(4)}}(z)=I+O\left(e^{-c_{2}|a|^{3}}\right) \quad \text { as } a \rightarrow-\infty,
$$

uniformly for $z \in\left[i\left(-c^{*}+\delta\right), i\left(c^{*}-\delta\right)\right] \backslash\{0\}$.

\subsection{Construction of the global parametrix $M^{(\infty)}$}

If we suppress all entries of the jump matrices for $M^{(4)}$ that exponentially decay as $a \rightarrow-\infty$, we are led to the following RH problem for the global parametrix $M^{(\infty)}$.

RH problem 5.6 (Global parametrix). We look for a $4 \times 4$ matrix valued function $M^{(\infty)}$ that satisfies

(1) $M^{(\infty)}$ is analytic in $\mathbb{C} \backslash \Sigma_{M^{(\infty)}}$, where the contour $\Sigma_{M^{(\infty)}}$ consists of the real line oriented from left to right and the purely imaginary interval $[-i c, i c]$ oriented from bottom to top. 


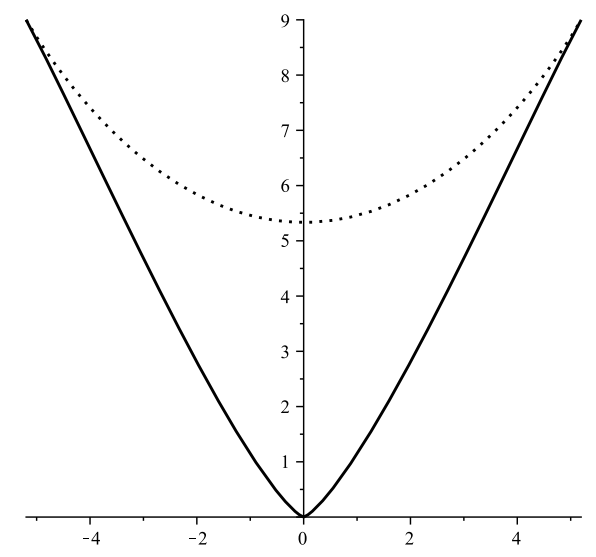

Figure 8: Plots of $\lambda_{4,+}^{*}(z)$ (solid) and $\lambda_{4,-}^{*}(z)$ (dashed) for $z \in\left(-i c^{*}, i c^{*}\right)$. Here $z=i x$ with $x$ on the horizontal axis.

(2) For $z \in \Sigma_{M^{(\infty)}}$, we have

$$
\begin{array}{ll}
M_{+}^{(\infty)}(x)=M_{-}^{(\infty)}(x)\left(\begin{array}{cccc}
0 & 0 & 1 & 0 \\
0 & 1 & 0 & 0 \\
-1 & 0 & 0 & 0 \\
0 & 0 & 0 & 1
\end{array}\right), & \text { for } x \in(0, \infty), \\
M_{+}^{(\infty)}(x)=M_{-}^{(\infty)}(x)\left(\begin{array}{cccc}
1 & 0 & 0 & 0 \\
0 & 0 & 0 & 1 \\
0 & 0 & 1 & 0 \\
0 & -1 & 0 & 0
\end{array}\right), & \text { for } x \in(-\infty, 0), \\
M_{+}^{(\infty)}(z)=M_{-}^{(\infty)}(z)\left(\begin{array}{cccc}
1 & 0 & 0 & 0 \\
0 & 1 & 0 & 0 \\
0 & 0 & 0 & 1 \\
0 & 0 & -1 & 0
\end{array}\right), & \text { for } z \in(-i c, 0) \cup(0, i c) .
\end{array}
$$

(3) As $z \rightarrow \infty$, the following asymptotic formula holds

$$
M^{(\infty)}(z)=\left(I+\mathcal{O}\left(z^{-1}\right)\right) B(z) A .
$$

(4) We have

$$
M^{(\infty)}(z)=\mathcal{O}\left(z^{-1 / 3}\right), \quad \text { as } z \rightarrow 0
$$

and

$$
M^{(\infty)}(z)=\mathcal{O}\left((z \mp i c)^{-1 / 4}\right), \quad \text { as } z \rightarrow \pm i c .
$$

An explicit solution of this $\mathrm{RH}$ problem can be built out of the $w$-functions introduced in Lemma 4.1. 


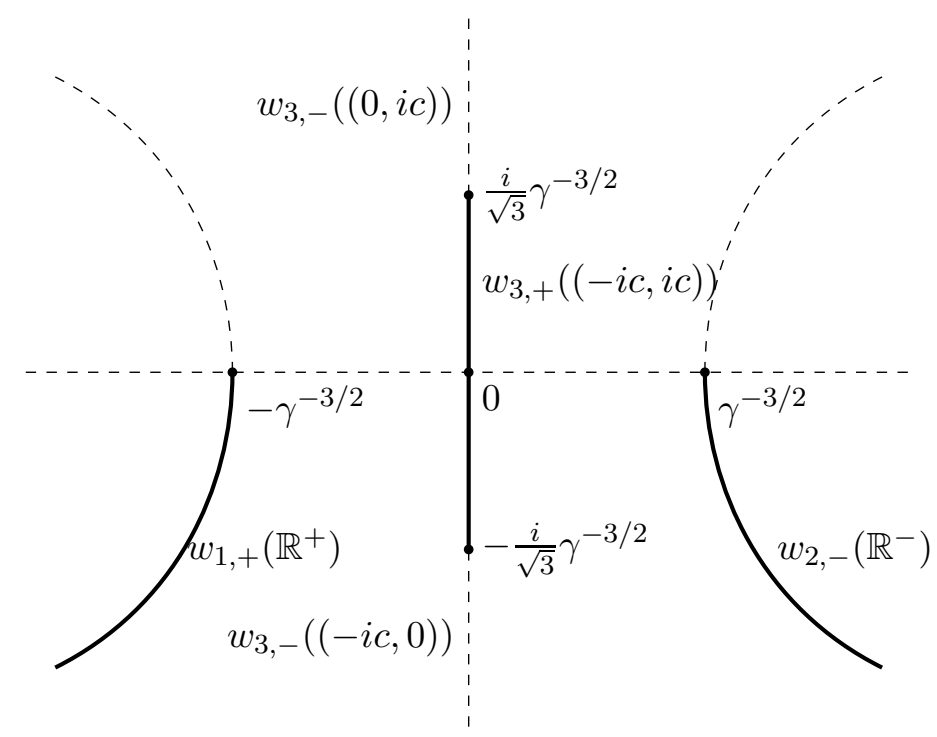

Figure 9: Image of the map $w: \mathcal{R} \mapsto \overline{\mathbb{C}}$. The solid and dashed lines are the images of the cuts in the Riemann surface $\mathcal{R}$ under this map. The solid lines represent the branch cut of the function $\kappa(w)$ defined in (5.23).

Lemma 5.7. With the functions $w_{j}, j=1,2,3,4$, defined in Lemma 4.1, the global parametrix $M^{(\infty)}$ is explicitly given by

$$
M^{(\infty)}(z)=\left(\begin{array}{llll}
F_{1}\left(w_{1}(z)\right) & F_{1}\left(w_{2}(z)\right) & F_{1}\left(w_{3}(z)\right) & F_{1}\left(w_{4}(z)\right) \\
F_{2}\left(w_{1}(z)\right) & F_{2}\left(w_{2}(z)\right) & F_{2}\left(w_{3}(z)\right) & F_{2}\left(w_{4}(z)\right) \\
F_{3}\left(w_{1}(z)\right) & F_{3}\left(w_{2}(z)\right) & F_{3}\left(w_{3}(z)\right) & F_{3}\left(w_{4}(z)\right) \\
F_{4}\left(w_{1}(z)\right) & F_{4}\left(w_{2}(z)\right) & F_{4}\left(w_{3}(z)\right) & F_{4}\left(w_{4}(z)\right)
\end{array}\right)
$$

where

$$
\begin{array}{ll}
F_{1}(w)=-\frac{\gamma^{15 / 8}}{\sqrt{6} \kappa(w)}\left(w+\gamma^{-3 / 2}\right)\left(w-\gamma^{-3 / 2}\right)^{2}, & F_{3}(w)=-\frac{i \gamma^{-3 / 8}}{2 \sqrt{6} \kappa(w)}\left(w-\gamma^{-3 / 2}\right)^{2}, \\
F_{2}(w)=\frac{\gamma^{15 / 8}}{\sqrt{6} \kappa(w)}\left(w+\gamma^{-3 / 2}\right)^{2}\left(w-\gamma^{-3 / 2}\right), & F_{4}(w)=\frac{i \gamma^{-3 / 8}}{2 \sqrt{6} \kappa(w)}\left(w+\gamma^{-3 / 2}\right)^{2},
\end{array}
$$

and

$$
\kappa(w)=\left(\left(w^{2}-\gamma^{-3}\right)\left(w^{2}+\frac{1}{3} \gamma^{-3}\right)\right)^{1 / 2}
$$

is defined in the $w$-plane with branch cut along $w_{1,+}\left(\mathbb{R}^{+}\right) \cup w_{3,+}((-i c, i c)) \cup w_{2,+}\left(\mathbb{R}^{-}\right)$ (see Figure 9) such that

$$
\kappa(w)=w^{2}(1+\mathcal{O}(1 / w)),
$$

as $w \rightarrow+\infty$. 
Proof. The jump condition follows from item (a) in Lemma 4.1 and (5.22). A straightforward calculation with the aid of item (c), (d), and (e) in Lemma 4.1 leads to the asymptotic behavior of $M^{(\infty)}(z)$ in (5.19), (5.20) and (5.21).

The global parametrix $M^{(\infty)}$ is a good approximation for $M^{(4)}$ for values of $z$ bounded away from 0 and $\pm i c^{*}$. We will construct local parametrices near these points in the next two sections.

\subsection{Construction of the local parametrices around $\pm i c^{*}$}

In the disks $D\left( \pm i c^{*}, \delta\right)$ around $\pm i c^{*}$ we build local parametrices $M^{( \pm i c)}$ respectively. The idea is to construct $M^{( \pm i c)}$ such that it makes exactly the same jumps in the disk $D\left( \pm i c^{*}, \delta\right)$ as $M^{(4)}$, and satisfies

$$
M^{( \pm i c)}(z)=\left(I+\mathcal{O}\left(|a|^{-3}\right)\right) M^{(\infty)}(z), \quad \text { uniformly for } z \in \partial D\left(\mp i c^{*}, \delta\right) \backslash \Sigma_{M^{(4)}},
$$

as $a \rightarrow-\infty$. This construction can be done using Airy functions and their derivatives. As this construction is standard (cf. [18, 20]) and the explicit formulas are irrelevant to the proofs of our main theorems, we will omit it here.

\subsection{Construction of the local parametrix around the origin}

In this section we construct a local parametrix $M^{(0)}$ in the disk $D(0, \delta)$ based on the Pearcey parametrix; see RH problem $[5.10$ below. First, we observe that the $(4,4)$ entry of the jump for $M^{(4)}$ on the imaginary axis is exponentially small, uniformly in a neighborhood of the origin; see item (b) of Corollary 5.5. Therefore, we may ignore this entry in the construction of the local parametrix around the origin which then has to satisfy the following conditions.

RH problem 5.8 (Local parametrix around the origin). We look for a $4 \times 4$ matrix valued function $M^{(0)}: D(0, \delta) \backslash \Sigma_{M^{(4)}} \rightarrow \mathbb{C}$ satisfying

(1) $M^{(0)}$ is analytic on $D(0, \delta) \backslash \Sigma_{M^{(4)}}$, where $\Sigma_{M^{(4)}}=\Sigma_{M^{(3)}}$ is defined in (5.7).

(2) For $z \in D(0, \delta) \cap \Sigma_{M^{(4)}}$, we have

$$
M_{+}^{(0)}(z)=M_{-}^{(0)}(z) J^{(0)}(z),
$$

with $J^{(0)}$ as indicated in Figure 10.

(3) As $a \rightarrow-\infty$, the asymptotic formula

$$
M^{(0)}(z)=\left(I+\mathcal{O}\left(|a|^{-3 / 2}\right)\right) M^{(\infty)}(z)
$$

uniformly holds for $z \in \partial D(0, \delta) \backslash \Sigma_{M^{(4)}}$.

(4) $M^{(0)}$ is bounded near the origin. 


$$
\left(\begin{array}{cccc}
1 & -e^{|a|^{3}\left(\lambda_{2}-\lambda_{1}\right)} & 0 & 0 \\
0 & 1 & 0 & 0 \\
0 & 0 & 1 & 0 \\
0 & e^{|a|^{3}\left(\lambda_{2}-\lambda_{4}\right)} & 0 & 1
\end{array}\right)\left(\begin{array}{cccc}
1 & 0 & 0 & 0 \\
0 & 1 & 0 & 0 \\
0 & 0 & 0 & 1 \\
0 & 0 & -1 & 0
\end{array}\right)\left(\begin{array}{cccc}
1 & 0 & 0 & 0 \\
-e^{|a|^{3}\left(\lambda_{1}-\lambda_{2}\right)} & 1 & 0 & 0 \\
e^{|a|^{3}\left(\lambda_{1}-\lambda_{3}\right)} & 0 & 1 & 0 \\
0 & 0 & 0 & 1
\end{array}\right)
$$

Figure 10: The jump contour and jump matrices $J^{(0)}$ for the local parametrix $M^{(0)}$ around zero. On the circle the matching condition (5.25) is imposed.

In a first step to solve the $\mathrm{RH}$ problem [5.8, we make a transformation that reduces the jumps of $M^{(0)}$ to constant matrices. Moreover we want the jumps on the imaginary axis to disappear. To this end we define

$$
\widetilde{M}^{(0)}(z)=M^{(0)}(z) \operatorname{diag}\left(e^{-|a|^{3} \lambda_{1}(z)}, e^{-|a|^{3} \lambda_{2}(z)}, e^{-|a|^{3} \lambda_{3}(z)}, e^{-|a|^{3} \lambda_{4}(z)}\right) C_{ \pm}^{-1},
$$

for $\pm \operatorname{Re} z>0$, where $C_{ \pm}$are two constant matrices given by

$$
C_{+}=I_{4}, \quad C_{-}=\operatorname{diag}\left(1,1,\left(\begin{array}{cc}
0 & 1 \\
-1 & 0
\end{array}\right)\right) .
$$

A straightforward check shows that the jumps for $\widetilde{M}^{(0)}$, as indicated in Figure 11, are indeed constant. Furthermore, all jump matrices have the block form $\left(\begin{array}{cc}Q & 0 \\ 0 & 1\end{array}\right)$, where $Q$ is a non-trivial $3 \times 3$ constant matrix. Combined with the asymptotic behavior of the $\lambda$-functions around the origin (see items (b) and (d) in Lemma 4.2), this leads us to propose the following model $\mathrm{RH}$ problem.

RH problem 5.9 (Model RH problem for the local parametrix around the origin). We look for a $3 \times 3$ matrix valued function $\Phi(\cdot ; \rho)$ depending on a parameter $\rho \in \mathbb{R}$ that satisfies the following conditions.

(1) $\Phi$ is defined and analytic on $\mathbb{C} \backslash \Sigma_{\Phi}$, where the contour $\Sigma_{\Phi}$ consists of 6 semi-infinite rays as shown in Figure 12. 


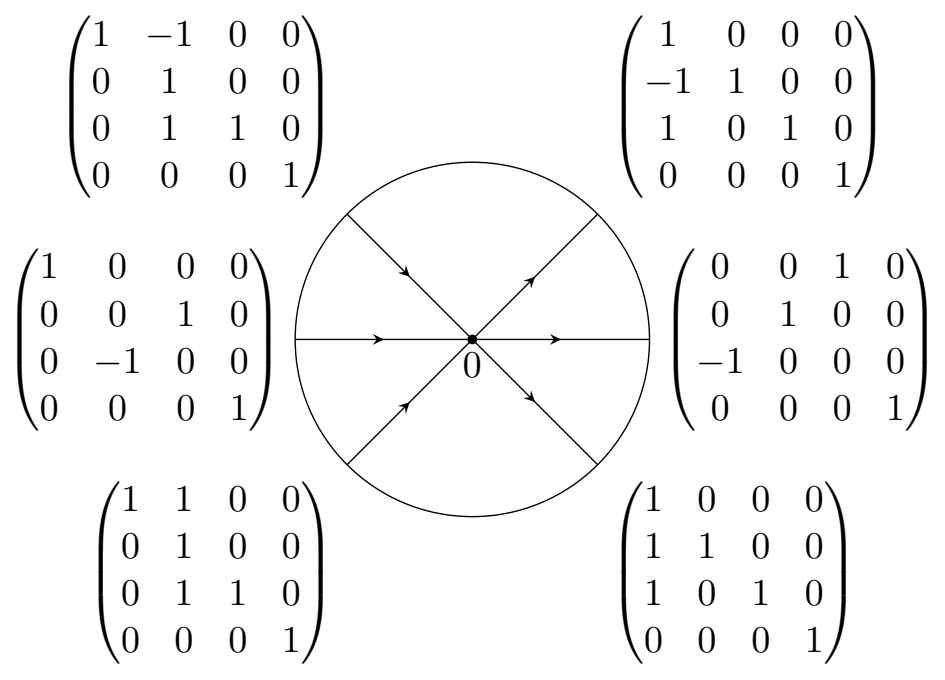

Figure 11: The jump contour and jump matrices for $\widetilde{M}^{(0)}$ around zero.

(2) For $z \in \Sigma_{\Phi}$, we have

$$
\Phi_{+}(z)=\Phi_{-}(z) J_{\Phi}(z),
$$

where the jump matrix $J_{\Phi}$ is constant on each ray and specified in Figure 12.

(3) As $z \rightarrow \infty$ and $\pm \operatorname{Im} z>0$, we have

$$
\Phi(z)=\sqrt{\frac{1}{6 \pi}} i e^{-\rho^{2} / 8} \operatorname{diag}\left(z^{1 / 3}, 1, z^{-1 / 3}\right) L_{ \pm}\left(I+\mathcal{O}\left(z^{-2 / 3}\right)\right) e^{\Theta(z ; \rho)},
$$

where $L_{ \pm}$are constant matrices

$$
L_{+}=\left(\begin{array}{ccc}
-\omega & -1 & -\omega^{2} \\
-1 & -1 & -1 \\
-\omega^{2} & -1 & -\omega
\end{array}\right), \quad L_{-}=\left(\begin{array}{ccc}
-\omega^{2} & -1 & \omega \\
-1 & -1 & 1 \\
-\omega & -1 & \omega^{2}
\end{array}\right)
$$

and $\Theta(z ; \rho)$ is given by

$$
\Theta(z ; \rho)= \begin{cases}\operatorname{diag}\left(-\theta_{2}(z ; \rho),-\theta_{3}(z ; \rho),-\theta_{1}(z ; \rho)\right), & \text { for } \operatorname{Im} z>0 \\ \operatorname{diag}\left(-\theta_{1}(z ; \rho),-\theta_{3}(z ; \rho),-\theta_{2}(z ; \rho)\right), & \text { for } \operatorname{Im} z<0\end{cases}
$$

with

$$
\theta_{k}(z ; \rho)=\frac{3}{4} \omega^{2 k} z^{4 / 3}+\frac{\rho}{2} \omega^{k} z^{2 / 3}, \quad k=1,2,3 .
$$

(4) $\Phi(z)$ is bounded near the origin.

At this moment it is not clear where the exact formulation of the asymptotics in item (3) comes from. This follows a posteriori from the solution of the $\mathrm{RH}$ problem given in Lemma 5.11. 


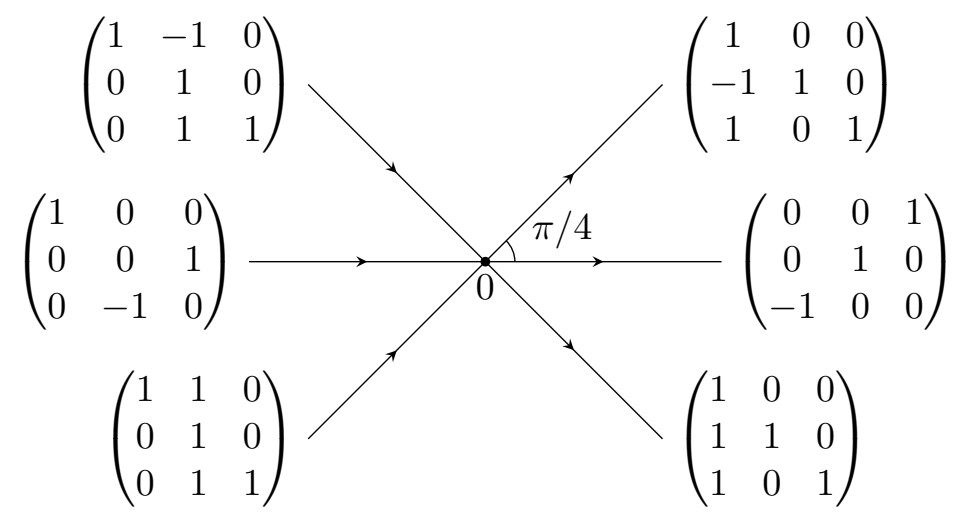

Figure 12: The jump contour $\Sigma_{\Phi}$ and jump matrices for the model RH problem $\Phi$.

Note that $\Phi$ depends on the parameter $\rho$ through the asymptotics (5.28). It turns out that we can solve this model RH problem using the Pearcey parametrix that was introduced by Bleher and Kuijlaars in the context of random matrices with external source [12]. The Pearcey parametrix is the unique solution to the following RH problem.

RH problem $\mathbf{5 . 1 0}$ (Pearcey parametrix).

(1) $\Phi^{\mathrm{Pe}}(\cdot ; \rho)$ is a $3 \times 3$ matrix valued function depending a parameter $\rho \in \mathbb{R}$, defined and analytic on $\mathbb{C} \backslash \Sigma_{\Phi}$.

(2) For $z \in \Sigma_{\Phi}^{\mathrm{Pe}}$, the function $\Phi^{\mathrm{Pe}}$ has the jump

$$
\Phi_{+}^{\mathrm{Pe}}(z)=\Phi_{-}^{\mathrm{Pe}}(z) J_{\Phi}^{\mathrm{Pe}}(z),
$$

where the jump matrix $J_{\Phi}^{\mathrm{Pe}}$ is constant on each ray and specified in Figure 13.

(3) As $z \rightarrow \infty$ and $\pm \operatorname{Im} z>0$, we have

$$
\Phi^{\mathrm{Pe}}(z)=\sqrt{\frac{2 \pi}{3}} i e^{\rho^{2} / 8} \operatorname{diag}\left(z^{-1 / 3}, 1, z^{1 / 3}\right) L_{ \pm}^{\mathrm{Pe}}\left(I+\mathcal{O}\left(z^{-2 / 3}\right)\right) e^{\Theta(z ; \rho)},
$$

where $L_{ \pm}^{\mathrm{Pe}}$ are constant matrices

$$
L_{+}^{\mathrm{Pe}}=\left(\begin{array}{ccc}
-\omega & \omega^{2} & 1 \\
-1 & 1 & 1 \\
-\omega^{2} & \omega & 1
\end{array}\right), \quad L_{-}^{\mathrm{Pe}}=\left(\begin{array}{ccc}
\omega^{2} & \omega & 1 \\
1 & 1 & 1 \\
\omega & \omega^{2} & 1
\end{array}\right)
$$

and $\Theta(z)$ given by

$$
\Theta(z ; \rho)= \begin{cases}\operatorname{diag}\left(\theta_{1}(z ; \rho), \theta_{2}(z ; \rho), \theta_{3}(z ; \rho)\right), & \text { for } \operatorname{Im} z>0, \\ \operatorname{diag}\left(\theta_{2}(z ; \rho), \theta_{1}(z ; \rho), \theta_{3}(z ; \rho)\right), & \text { for } \operatorname{Im} z<0,\end{cases}
$$

and where $\theta_{k}(z ; \rho)$ is defined in (5.30). 


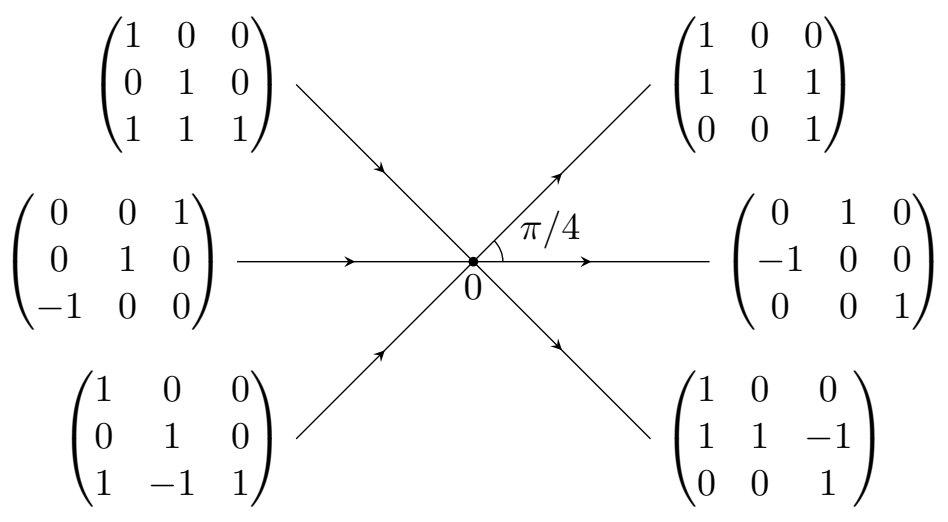

Figure 13: The jump contour and jump matrices for the Pearcey parametrix $\Phi^{\mathrm{Pe}}$.

(4) $\Phi^{\mathrm{Pe}}(z)$ is bounded near the origin.

The above RH problem has a unique solution $\Phi^{\mathrm{Pe}}$, given in terms of solutions of the Pearcey differential equation

$$
y^{\prime \prime \prime}(z)-\rho p^{\prime}(z)-z y(z)=0 .
$$

Since the exact formula of $\Phi^{\mathrm{Pe}}$ is not relevant for our purposes, we decide not to write it down and refer to [12, Section 8.1] for details.

The next lemma states that $\mathrm{RH}$ problem 5.9 can be solved in terms of the Pearcey parametrix.

Lemma 5.11. Let $\Phi^{\mathrm{Pe}}$ be the Pearcey parametrix as given in $R H$ problem 5.10 , the solution of $R H$ problem 5.9 is given by

$$
\Phi(z)=\Phi^{\mathrm{Pe}}(z)^{-T}\left(\begin{array}{ccc}
0 & 0 & -1 \\
1 & 0 & 0 \\
0 & 1 & 0
\end{array}\right),
$$

where the superscript ${ }^{-T}$ stands for inverse transpose.

Proof. It is straightforward to check that the right-hand side of (5.33) satisfies RH problem 5.9. Then (5.33) follows from the uniqueness of the solution to this RH problem.

Now, we are ready to construct the local parametrix $M^{(0)}$. In view of $(\underline{5.26})$, we look for a parametrix in the form

$$
\begin{aligned}
M^{(0)}(z)=E_{0}(z) & \left(\begin{array}{cc}
\Phi(f(z) ; \rho(z)) & 0 \\
0 & \phi(z)
\end{array}\right) C_{ \pm} \\
& \times \operatorname{diag}\left(e^{|a|^{3} \lambda_{1}(z)}, e^{|a|^{3} \lambda_{2}(z)}, e^{|a|^{3} \lambda_{3}(z)}, e^{|a|^{3} \lambda_{4}(z)}\right), \quad \pm \operatorname{Re} z>0,
\end{aligned}
$$


where $E_{0}(z)$ is an analytic prefactor, $f(z)$ is a conformal map near the origin, $\phi(z)$ is an analytic function and $C_{ \pm}$are the constant matrices shown in (5.27). The parameter $\rho$ will also be chosen to be dependent on $z$.

Recall the functions $G(z), H(z)$, and $K(z)$ introduced in item $(d)$ of Lemma 4.2, We first define

$$
f(z)=f(z ; a)=|a|^{9 / 4}\left(\frac{4}{3} H(z)\right)^{3 / 4} z, \quad z \in D(0, \delta) .
$$

By (4.12), it is readily seen that

$$
f(z)=|a|^{9 / 4}\left(\frac{4}{3} H(0)\right)^{3 / 4} z+\mathcal{O}\left(z^{3}\right)
$$

as $z \rightarrow 0$. Hence, $f$ is a conformal map in a neighborhood of the origin. We then modify the contours $\left(\Sigma_{M^{(4)}} \backslash i \mathbb{R}\right) \cap D(0, \delta)$ if necessary in such a way that $f$ maps them into $\Sigma_{\Phi}$. Next we set

$$
\rho(z)=\rho(z ; a)=2|a|^{3} \frac{G(z) z^{2 / 3}}{f(z)^{2 / 3}}, \quad z \in D(0, \delta)
$$

and

$$
\phi(z)=\phi(z ; a)= \begin{cases}e^{-|a|^{3} \lambda_{4}(z)}, & \operatorname{Re} z>0, \\ e^{-|a|{ }^{3} \lambda_{3}(z)}, & \operatorname{Re} z<0 .\end{cases}
$$

Also the function $\phi(z)$ is analytic on $D(0, \delta)$, which follows from (4.10). With these definitions of $f(z)$ and $\rho(z)$, it is easily seen from items (b) and (d) in Lemma 4.2 that

$$
\begin{aligned}
& \theta_{1}(f(z) ; \rho(z))= \begin{cases}|a|^{3}\left(\lambda_{3}(z)-K(z) z^{2}\right), & \text { for } z \in D(0, \delta) \cap I, \\
|a|^{3}\left(\lambda_{4}(z)-K(z) z^{2}\right), & \text { for } z \in D(0, \delta) \cap I I, \\
|a|^{3}\left(\lambda_{1}(z)-K(z) z^{2}\right), & \text { for } z \in D(0, \delta) \cap(I I I \cup I V),\end{cases} \\
& \theta_{2}(f(z) ; \rho(z))= \begin{cases}|a|^{3}\left(\lambda_{1}(z)-K(z) z^{2}\right), & \text { for } z \in D(0, \delta) \cap(I \cup I I), \\
|a|^{3}\left(\lambda_{4}(z)-K(z) z^{2}\right), & \text { for } z \in D(0, \delta) \cap I I I, \\
|a|^{3}\left(\lambda_{3}(z)-K(z) z^{2}\right), & \text { for } z \in D(0, \delta) \cap I V,\end{cases} \\
& \theta_{3}(f(z) ; \rho(z))=|a|^{3}\left(\lambda_{2}(z)-K(z) z^{2}\right),
\end{aligned}
$$

Finally, the matching condition (5.25) leads us to the definition of the prefactor $E_{0}(z)$ as

$$
\begin{aligned}
& E_{0}(z)=M^{(\infty)}(z)\left(C_{ \pm}\right)^{-1} \\
& \quad \times\left(\begin{array}{cc}
-i \sqrt{6 \pi} e^{\rho(z)^{2} / 8-|a|^{3} K(z) z^{2}}\left(L_{ \pm}\right)^{-1} \operatorname{diag}\left(f(z)^{-1 / 3}, 1, f(z)^{1 / 3}\right) & 0 \\
0 & 1
\end{array}\right), \quad \pm \operatorname{Re} z>0
\end{aligned}
$$

where $L_{ \pm}$is defined in (5.29). We then have the following lemma. 
Lemma 5.12. $E_{0}(z)$ has an analytic continuation to $D(0, \delta)$.

Proof. $E_{0}(z)$ is clearly analytic in $D(0, \delta) \backslash(\mathbb{R} \cup i \mathbb{R})$. For $z \in i \mathbb{R} \backslash\{0\}$ we have that $E_{0,+}(z)=E_{0,-}(z)$ as a consequence of (5.18) and (5.27). There is no jump on $\mathbb{R}^{+}$ either, because of (5.16) and the observation

$$
L_{+}=L_{-}\left(\begin{array}{ccc}
0 & 0 & -1 \\
0 & 1 & 0 \\
1 & 0 & 0
\end{array}\right)
$$

With a bit more effort one can also check that the jump on $\mathbb{R}^{-}$is trivial. Hence $E_{0}(z)$ is analytic in the punctured disk $D(0, \delta) \backslash\{0\}$. It follows from the behavior of the global parametrix $M^{(\infty)}$ around zero that the singularity of $E_{0}(z)$ at the origin is removable.

It is now straightforward to check that the jumps of $M^{(0)}$ on $D(0, \delta) \cap \Sigma_{M^{(4)}}$ and its large $|a|$ behavior on $\partial D(0, \delta)$ (with the aid of (5.28) and (5.37)) are indeed of our required form. In summary, we have the following lemma.

Lemma 5.13. The matrix valued function $M^{(0)}$ defined in (5.34) satisfies conditions (1)-(4) of RH problem 5.8.

\subsection{Final transformation: $M^{(4)} \mapsto M^{(5)}$}

Using the global parametrix $M^{(\infty)}$ and the local parametrices $M^{( \pm i c)}$ and $M^{(0)}$, we define the fifth transformation $M^{(4)} \mapsto M^{(5)}$ as follows

$$
M^{(5)}(z)= \begin{cases}M^{(4)}(z)\left(M^{(\infty)}(z)\right)^{-1} & \text { for } z \in \mathbb{C} \backslash\left(\Sigma_{M^{(4)}} \cup D(0, \delta) \cup D\left( \pm i c^{*}, \delta\right)\right), \\ M^{(4)}(z)\left(M^{(0)}(z)\right)^{-1} & \text { for } z \in D(0, \delta) \backslash \Sigma_{M^{(4)}}, \\ M^{(4)}(z)\left(M^{( \pm i c)}(z)\right)^{-1} & \text { for } z \in D\left( \pm i c^{*}, \delta\right) \backslash \Sigma_{M^{(4)}} .\end{cases}
$$

Then $M^{(5)}$ is defined and analytic outside of $\Sigma_{M^{(4)}}$ and the three disks around 0 and $\pm i c^{*}$, with an analytic continuation across those parts of $\Sigma_{M^{(4)}}$ where the jumps of the parametrices coincide with those of $M^{(4)}$. What remains are the jumps on a contour $\Sigma_{M^{(5)}}$ that consists of the three circles around $0, \pm i c^{*}$, the parts of $\Gamma_{1}, \widetilde{\Gamma}_{2}, \widetilde{\Gamma}_{3}, \Gamma_{4}, \Gamma_{6}$, $\widetilde{\Gamma}_{7}, \widetilde{\Gamma}_{8}$ and $\Gamma_{9}$ outside of the disks, and $\left(i\left(-c^{*}+\delta\right), i\left(c^{*}-\delta\right)\right)$. The circles are oriented clockwise. Then $M^{(5)}$ satisfies the following RH problem.

\section{RH problem 5.14.}

(1) $M^{(5)}$ is defined and analytic in $\mathbb{C} \backslash \Sigma_{M^{(5)}}$.

(2) For $z \in \Sigma_{M}^{(5)}$, we have

$$
M_{+}^{(5)}(z)=M_{-}^{(5)}(z) J_{M^{(5)}}(z)
$$


where

$$
J_{M^{(5)}}(z)= \begin{cases}M^{(0)}(z)\left(M^{(\infty)}(z)\right)^{-1} & \text { for } z \in \partial D(0, \delta), \\ M^{( \pm i c)}(z)\left(M^{(\infty)}(z)\right)^{-1} & \text { for } z \in \partial D\left( \pm i c^{*}, \delta\right), \\ M_{-}^{(0)}(z) J_{M^{(4)}}(z)\left(M_{+}^{(0)}(z)\right)^{-1} & \text { for } z \in(-i \delta, i \delta), \\ M_{-}^{(\infty)}(z) J_{M^{(4)}}(z)\left(M_{+}^{(\infty)}(z)\right)^{-1} & \text { elsewhere on } \Sigma_{M^{(5)}}\end{cases}
$$

(3) As $z \rightarrow \infty$, we have

$$
M^{(5)}(z)=I+O(1 / z)
$$

Furthermore, the jump matrix $J_{M^{(5)}}$ tends to the identity matrix on $\Sigma_{M^{(5)}}$ as $a \rightarrow$ $-\infty$, both uniformly and in $L^{2}$-sense. Indeed, from the matching conditions (5.24) and (5.25), we see that

$$
J_{M^{(5)}}(z)=I+\mathcal{O}\left(|a|^{-3 / 2}\right)
$$

as $a \rightarrow-\infty$, uniformly on the circles around 0 and $\pm i c^{*}$. On the remaining parts of $\Sigma_{M^{(5)}}, J_{M^{(5)}}$ is uniformly exponentially small (see Corollary [5.5). Then, as in [19, 20], we conclude that

$$
M^{(5)}(z)=I+O\left(\frac{1}{|a|^{3 / 2}(|z|+1)}\right),
$$

as $a \rightarrow-\infty$, uniformly for $z$ in the complex plane outside of $\Sigma_{M^{(5)}}$.

The estimate (5.41) is the main outcome of our Deift-Zhou steepest descent analysis for $\mathrm{RH}$ problem 1.1. We will use it to prove our main results in the next section.

\section{Proofs of the main theorems}

\subsection{RH formula for the Pearcey kernel}

Before coming to the proofs of the main theorems, we state the following lemma giving an expression of the Pearcey kernel in terms of the Pearcey parametrix. 
Lemma 6.1. Let $K^{\mathrm{Pe}}(x, y ; \rho)$ be the Pearcey kernel defined in (2.8), we have

$$
K^{\mathrm{Pe}}(x, y ; \rho)= \begin{cases}\frac{1}{2 \pi i(x-y)}\left(\begin{array}{lll}
-1 & 1 & 0
\end{array}\right) \Phi_{+}^{\mathrm{Pe}}(y ; \rho)^{-1} \Phi_{+}^{\mathrm{Pe}}(x ; \rho)\left(\begin{array}{l}
1 \\
1 \\
0
\end{array}\right), & \text { for } x, y>0, \\
\frac{1}{2 \pi i(x-y)}\left(\begin{array}{lll}
-1 & 0 & 1
\end{array}\right) \Phi_{+}^{\mathrm{Pe}}(y ; \rho)^{-1} \Phi_{+}^{\mathrm{Pe}}(x ; \rho)\left(\begin{array}{l}
1 \\
1 \\
0
\end{array}\right), & \text { for } x>0, y<0, \\
\frac{1}{2 \pi i(x-y)}\left(\begin{array}{lll}
-1 & 1 & 0
\end{array}\right) \Phi_{+}^{\mathrm{Pe}}(y ; \rho)^{-1} \Phi_{+}^{\mathrm{Pe}}(x ; \rho)\left(\begin{array}{l}
1 \\
0 \\
1
\end{array}\right), & \text { for } x<0, y>0, \\
\frac{1}{2 \pi i(x-y)}\left(\begin{array}{lll}
-1 & 0 & 1
\end{array}\right) \Phi_{+}^{\mathrm{Pe}}(y ; \rho)^{-1} \Phi_{+}^{\mathrm{Pe}}(x ; \rho)\left(\begin{array}{l}
1 \\
0 \\
1
\end{array}\right), & \text { for } x, y<0,\end{cases}
$$

where $\Phi^{\mathrm{Pe}}(\cdot ; \rho)$ is the the unique solution of $R H$ problem 5.10 .

Proof. See [12, Section 10.2].

\subsection{Proof of Theorem 2.3}

First we prove (2.10). We will focus on the case $u, v>0$, or equivalently, $x, y>0$. Other cases can be proved similarly. We start from (2.6), and the strategy is to express this kernel in terms of $M^{(5)}$ instead of $M$ by unfolding all transformations $M \mapsto M^{(1)} \mapsto$ $M^{(2)} \mapsto M^{(3)} \mapsto M^{(4)} \mapsto M^{(5)}$ of the steepest descent analysis.

From the first transformation $M \mapsto M^{(1)}$ in (5.2), it follows

$$
\begin{aligned}
& a^{2} K^{\operatorname{tac}}\left(a^{2} u, a^{2} v ;-\frac{1}{2} a^{2},|a|\left(1+\frac{\sigma}{2|a|^{3 / 2}}\right)\right) \\
& =\frac{1}{2 \pi i(u-v)}\left(\begin{array}{cccc}
-1 & 0 & 1 & 0
\end{array}\right) M_{+}^{(1)}(v ; a)^{-1} M_{+}^{(1)}(u ; a)\left(\begin{array}{l}
1 \\
0 \\
1 \\
0
\end{array}\right)
\end{aligned}
$$

where, since we work in Brownian paths model, we chose the top sign in $\pm \sigma$. The transformations $M^{(1)} \mapsto M^{(2)} \mapsto M^{(3)}$ in (5.5) and (5.6) leave this formula essentially 
unaffected. Applying the transformation $M^{(3)} \mapsto M^{(4)}$ in (5.8), however, yields

$$
\begin{aligned}
& a^{2} K^{\mathrm{tac}}\left(a^{2} u, a^{2} v ; a,-\frac{1}{2} a^{2},|a|\left(1+\frac{\sigma}{2|a|^{3 / 2}}\right)\right) \\
& =\frac{1}{2 \pi i(u-v)}\left(\begin{array}{llll}
-e^{|a|^{3} \lambda_{1,+}(v)} & 0 & e^{|a|^{3} \lambda_{3,+}(v)} & 0
\end{array}\right) M_{+}^{(4)}(v ; a)^{-1} M_{+}^{(4)}(u ; a)\left(\begin{array}{c}
e^{-|a|^{3} \lambda_{1,+}(u)} \\
0 \\
e^{-|a|^{3} \lambda_{3,+}(u)} \\
0
\end{array}\right) .
\end{aligned}
$$

In the next step we unfold the transformation $M^{(4)} \mapsto M^{(5)}$ in (5.39). Assuming that $0<u, v<\delta$, it follows from (5.34) that

$$
\begin{aligned}
& a^{2} K^{\operatorname{tac}}\left(a^{2} u, a^{2} v ;-\frac{1}{2} a^{2},|a|\left(1+\frac{\sigma}{2|a|^{3 / 2}}\right)\right)=\frac{1}{2 \pi i(u-v)} \\
& \times\left(\begin{array}{llll}
-1 & 0 & 1 & 0
\end{array}\right)\left(\begin{array}{cc}
\Phi(f(v) ; \rho(v))^{-1} & 0 \\
0 & \phi(v)^{-1}
\end{array}\right) E_{0}(v)^{-1} M^{(5)}(v)^{-1} \\
& \times M^{(5)}(u) E_{0}(u)\left(\begin{array}{cc}
\Phi(f(u) ; \rho(u)) & 0 \\
0 & \phi(u)
\end{array}\right)\left(\begin{array}{l}
1 \\
0 \\
1 \\
0
\end{array}\right) .
\end{aligned}
$$

Now we fix $x, y>0$ and take

$$
u=2^{-1 / 2}|a|^{-9 / 4} x, \quad v=2^{-1 / 2}|a|^{-9 / 4} y,
$$

so that $0<u, v<\delta$ for $|a|$ sufficiently large. Under this change of variables, it follows from (5.35) and (4.12) that

$$
f(u) \rightarrow x, \quad f(v) \rightarrow y,
$$

as $a \rightarrow-\infty$. Also (5.36), (5.35), (4.12), (4.11), and (4.5) imply

$$
\rho(u) \rightarrow \sigma, \quad \rho(v) \rightarrow \sigma,
$$

as $a \rightarrow-\infty$. Furthermore, by standard considerations it follows that

$$
M^{(5)}(v ; a)^{-1} M^{(5)}(u ; a)=I+\mathcal{O}\left(\frac{v-u}{|a|^{3 / 2}}\right)=I+\mathcal{O}\left(\frac{x-y}{|a|^{15 / 4}}\right),
$$

as $a \rightarrow-\infty$, uniformly for $x$ and $y$ in a compact subset of $\mathbb{R}$. Observe also that $E_{0}(u)=\mathcal{O}\left(|a|^{3 / 4}\right)$ as $a \rightarrow-\infty$; see (5.38) and (5.20). The same bound holds for $E_{0}(u)^{-1}, E_{0}(v)$ and $E_{0}(v)^{-1}$, so that we find

$$
E_{0}(v)^{-1} E_{0}(u)=I+\mathcal{O}\left(|u-v||a|^{3 / 2}\right)=I+\mathcal{O}\left(\frac{|x-y|}{|a|^{3 / 4}}\right) .
$$


Combining all these results, gives

$$
\begin{aligned}
\lim _{a \rightarrow-\infty} \frac{1}{\sqrt{2}|a|^{1 / 4}} K^{\operatorname{tac}}\left(\frac{x}{\sqrt{2}|a|^{1 / 4}}, \frac{y}{\sqrt{2}|a|^{1 / 4}} ;-\frac{1}{2} a^{2},|a|\left(1+\frac{\sigma}{2|a|^{3 / 2}}\right)\right) \\
=\frac{1}{2 \pi i(x-y)}\left(\begin{array}{ccc}
-1 & 0 & 1
\end{array}\right) \Phi(y ; \sigma)^{-1} \Phi(x ; \sigma)\left(\begin{array}{l}
1 \\
0 \\
1
\end{array}\right) .
\end{aligned}
$$

An appeal to (5.33) yields

$$
\begin{aligned}
& \lim _{a \rightarrow-\infty} \frac{1}{\sqrt{2}|a|^{1 / 4}} K^{\operatorname{tac}}\left(\frac{x}{\sqrt{2}|a|^{1 / 4}}, \frac{y}{\sqrt{2}|a|^{1 / 4}} ;-\frac{1}{2} a^{2},|a|\left(1+\frac{\sigma}{2|a|^{3 / 2}}\right)\right) \\
& =\frac{1}{2 \pi i(x-y)}\left(\begin{array}{lll}
1 & 1 & 0
\end{array}\right) \Phi^{\mathrm{Pe}}(y ; \sigma)^{T} \Phi^{\mathrm{Pe}}(x ; \sigma)^{-T}\left(\begin{array}{c}
1 \\
-1 \\
0
\end{array}\right) .
\end{aligned}
$$

Finally, by taking the transpose on both sides of the above formula, (2.10) follows from (6.1) for the case $x, y>0$.

Next we prove (2.11) from symmetry considerations. We start with the following symmetry relation

$$
M^{-T}(\zeta ; s, t)=\left(\begin{array}{cc}
0 & I_{2} \\
-I_{2} & 0
\end{array}\right) M(\zeta ; s,-t)\left(\begin{array}{cc}
0 & -I_{2} \\
I_{2} & 0
\end{array}\right)
$$

for $s, t \in \mathbb{R}$, which can be checked from RH problem 1.1. Here, $I_{2}$ denotes $2 \times 2$ identity matrix. Using (2.5) this leads to the following symmetry property of the tacnode kernel

$$
K^{\operatorname{tac}}(u, v ; s,-t)=K^{\operatorname{tac}}(v, u ; s, t) .
$$

Given this, (2.11) is immediate from (2.10).

This completes the proof of Theorem 2.3 .

\subsection{Proof of Theorem 3.3}

We start with a lemma that establishes a symmetry property of the Pearcey parametrix.

Lemma 6.2. Let $\Phi^{\mathrm{Pe}}(\cdot ; \rho)$ be the unique solution of $R H$ problem 5.10 . Then

$$
\operatorname{diag}(-i, 1, i) \Phi^{\mathrm{Pe}}(i z ;-\rho) B_{j}=\Phi^{\mathrm{Pe}}(z ; \rho), \quad \text { for } z \text { in the } j \text {-th quadrant, }
$$

where

$B_{1}=\left(\begin{array}{ccc}0 & 0 & -1 \\ -1 & 0 & 0 \\ 0 & 1 & 0\end{array}\right), \quad B_{2}=\left(\begin{array}{ccc}0 & 1 & 0 \\ -1 & 0 & 0 \\ 0 & 0 & 1\end{array}\right), \quad B_{3}=\left(\begin{array}{ccc}0 & 1 & 0 \\ 0 & 0 & 1 \\ 1 & 0 & 0\end{array}\right), \quad B_{4}=\left(\begin{array}{ccc}0 & 0 & -1 \\ 0 & 1 & 0 \\ 1 & 0 & 0\end{array}\right)$. 
Proof. We have to check that the left-hand side of (6.2) satisfies RH problem 5.10, It is straightforward to check the jump conditions in item (2). Checking the asymptotics is a bit more cumbersome. To that end the following observations are useful

$$
\begin{aligned}
& \theta_{1}(i z ;-\rho)= \begin{cases}\theta_{3}(z ; \rho), & \text { for } z \in I \cup I I I \cup I V, \\
\theta_{1}(z ; \rho), & \text { for } z \in I I,\end{cases} \\
& \theta_{2}(i z ;-\rho)= \begin{cases}\theta_{1}(z ; \rho), & \text { for } z \in I \cup I I I \cup I V, \\
\theta_{2}(z ; \rho), & \text { for } z \in I I,\end{cases} \\
& \theta_{3}(i z ;-\rho)= \begin{cases}\theta_{2}(z ; \rho), & \text { for } z \in I \cup I I I \cup I V, \\
\theta_{3}(z ; b), & \text { for } z \in I I,\end{cases}
\end{aligned}
$$

and also

$$
\begin{aligned}
e^{ \pm \pi i / 6}(i z)^{\mp 1 / 3} & =z^{\mp 1 / 3}, & & \text { for } z \in I \cup I I I \cup I V, \\
\mp i(i z)^{\mp 1 / 3} & =z^{\mp 1 / 3}, & & \text { for } z \in I I .
\end{aligned}
$$

We omit the details here.

The proof is similar to that of Theorem 2.3, and we will focus on the points where both proofs differ and leave some details to the reader. Note that we are now working in the context of the two-matrix model, so we choose the bottom sign in $\pm \sigma$; cf. (4.5) and (5.1).

Following the same ideas as in the proof of Theorem 2.3, we obtain

$$
\begin{aligned}
& a^{2} K^{\mathrm{cr}}\left(a^{2} u, a^{2} v ;-\frac{1}{2} a^{2},|a|\left(1-\frac{\sigma}{2|a|^{3 / 2}}\right)\right)=\frac{1}{2 \pi i(u-v)} \\
& \times\left(\begin{array}{llll}
-1 & 1 & 0 & 0
\end{array}\right)\left(\begin{array}{cc}
\Phi(f(i u) ; \rho(i u))^{-1} & 0 \\
0 & \phi(i u)^{-1}
\end{array}\right) E_{0}(i u)^{-1} M_{+}^{(5)}(i u)^{-1} \\
& \quad \times M_{+}^{(5)}(i v) E_{0}(i v)\left(\begin{array}{cc}
(f(i v) ; \rho(i v)) & 0 \\
0 & \phi(i v)
\end{array}\right)\left(\begin{array}{l}
1 \\
1 \\
0 \\
0
\end{array}\right),
\end{aligned}
$$

for $0<|u|,|v|<\delta$.

Now we fix $x, y \neq 0$ and take

$$
u=2^{-1 / 2}|a|^{-9 / 4} x, \quad v=2^{-1 / 2}|a|^{-9 / 4} y,
$$

so that $|u|,|v|<\delta$ for $|a|$ sufficiently large. Hence

$$
f(i u) \rightarrow i x, \quad f(i v) \rightarrow i y,
$$

as $a \rightarrow-\infty$ and

$$
\rho(i u) \rightarrow-\sigma, \quad \rho(i v) \rightarrow-\sigma,
$$


as $a \rightarrow-\infty$. Furthermore,

$$
M_{+}^{(5)}(i u ; a)^{-1} M_{+}^{(5)}(i v ; a)=I+\mathcal{O}\left(\frac{x-y}{|a|^{15 / 4}}\right)
$$

as $a \rightarrow-\infty$, uniformly for $x$ and $y$ in a compact subset of $\mathbb{R}$. Also

$$
E_{0}(i u)^{-1} E_{0}(i v)=I+\mathcal{O}\left(|u-v||a|^{3 / 2}\right)=I+\mathcal{O}\left(\frac{|x-y|}{|a|^{3 / 4}}\right) .
$$

These results, together with (5.33) and taking the transpose, imply

$$
\begin{aligned}
& \lim _{a \rightarrow-\infty} \frac{1}{\sqrt{2}|a|^{1 / 4}} K^{\mathrm{cr}}\left(\frac{x}{\sqrt{2}|a|^{1 / 4}}, \frac{y}{\sqrt{2}|a|^{1 / 4}} ;-\frac{1}{2} a^{2},|a|\left(1-\frac{\sigma}{2|a|^{3 / 2}}\right)\right) \\
& =\frac{1}{2 \pi i(x-y)}\left(\begin{array}{ccc}
0 & 1 & 1
\end{array}\right) \Phi^{\mathrm{Pe}}(i y ;-\sigma)^{-1} \Phi^{\mathrm{Pe}}(i x ;-\sigma)\left(\begin{array}{c}
0 \\
-1 \\
1
\end{array}\right) .
\end{aligned}
$$

Finally, we obtain Theorem 3.3 by applying Lemma 6.2 and (6.1) to the above formula.

\section{Acknowledgments}

We thank Steven Delvaux and Arno Kuijlaars for their careful reading of the manuscript and helpful comments. DG is a Research Assistant of the Fund for Scientific Research - Flanders (FWO), Belgium. LZ is a Postdoctoral Fellow of the Fund for Scientific Research - Flanders (FWO), Belgium.

\section{References}

[1] M. Abramowitz and I.A. Stegun, Handbook of Mathematical Functions, New York: Dover Publications, 1968.

[2] M. Adler, M. Cafasso and P. van Moerbeke, From the Pearcey to the Airy process, Electron. J. Probab. 16 (2011), 1048-1064.

[3] M. Adler, P. Ferrari and P. van Moerbeke, Non-intersecting random walks in the neighborhood of a symmetric tacnode, to appear in Ann. of Prob., arXiv:1007.1163

[4] M. Adler, K. Johansson and P. van Moerbeke, Double Aztec diamonds and the tacnode process, arXiv:1112.5532.

[5] M. Adler and P. van Moerbeke, PDEs for the Gaussian ensemble with external source and the Pearcey distribution, Comm. Pure Appl. Math. 60 (2007), 12611292. 
[6] M. Bertola and M. Cafasso, The transition between the gap probabilities from the Pearcey to the Airy process; a Riemann-Hilbert approach, Int. Math. Res. Not., doi:10.1093/imrn/rnr066 (2011).

[7] M. Bertola and M. Cafasso, Riemann-Hilbert approach to multi-time processes; the Airy and the Pearcey case, to appear in Phys. D, arXiv:1104.4940.

[8] M. Bertola, B.Eynard and J. Harnad, The PDEs of biorthogonal polynomials arising in the two-matrix model, Math. Phys. Anal. Geom. 9 (2006), 162-212.

[9] M. Bertola, B.Eynard and J. Harnad, Duality, biorthogonal polynomials and multimatrix models, Comm. Math. Phys. 229 (2002), 73-120.

[10] M. Bertola, B.Eynard and J. Harnad, Differential systems for biorthogonal polynomials appearing in 2-matrix models and the associated Riemann-Hilbert problem, Comm. Math. Phys. 243 (2003), 193-240.

[11] P. Bleher and A. Its, Double scaling limit in the random matrix model: the RiemannHilbert approach, Comm. Pure Appl. Math 56 (2003), 433-516

[12] P.M. Bleher and A.B.J. Kuijlaars, Large n limit of Gaussian random matrices with external source, part III: double scaling limit, Comm. Math. Phys. 270 (2007), 481517.

[13] E. Brézin and S. Hikami, Universal singularity at the closure of a gap in a random matrix theory, Phys. Rev. E. (3) 57 (1998), no. 4. 7176-7185.

[14] E. Brézin and S. Hikami, Level spacing of random matrices in an external source, Phys. Rev. E. (3) 58 (1998), no. 6, part A, 4140-4149.

[15] T. Claeys and A.B.J. Kuijlaars, Universality of the double scaling limit in random matrix models, Comm. Pure Appl. Math. 59 (2006), 1573-1603.

[16] T. Claeys, A.B.J. Kuijlaars and M. Vanlessen, Multi-critical unitary random matrix ensembles and the general Painlevé II equation, Ann. of Math. 168 (2008), 601-642.

[17] E. Daems, A.B.J. Kuijlaars and W. Veys, Asymptotics of non-intersecting Brownian motions and a $4 \times 4$ Riemann-Hilbert problem, J. Approx. Theory 153 (2008), 225256.

[18] P. Deift, Orthogonal Polynomials and Random Matrices: a Riemann-Hilbert approach, Courant Lecture Notes in Mathematics Vol. 3, Amer. Math. Soc., Providence R.I. 1999.

[19] P. Deift, T. Kriecherbauer, K. T-R. McLaughlin, S. Venakides and X. Zhou, Strong asymptotics of orthogonal polynomials with respect to exponential weights, Comm. Pure Appl. Math. 52 (1999), 1491-1552. 
[20] P. Deift, T. Kriecherbauer, K.T-R McLaughlin, S. Venakides and X. Zhou, Uniform asymptotics for polynomials orthogonal with respect to varying exponential weights and applications to universality questions in random matrix theory, Comm. Pure Appl. Math. 52 (1999), 1335-1425.

[21] P. Deift and X. Zhou, Asymptotics for the Painlevé II equation, Comm. Pure Appl. Math. 48 (1995), 277-337.

[22] S. Delvaux, Non-intersecting squared Bessel paths at a hard-edge tacnode, arXiv:1204.4430.

[23] S. Delvaux and A.B.J. Kuijlaars, A phase transition for non-intersecting Brownian motions, and the Painlevé II equation, Int. Math. Res. Not. (2009), 3639-3725.

[24] S. Delvaux, A.B.J. Kuijlaars and L. Zhang, Critical behavior of non-intersecting Brownian motions at a tacnode, Comm. Pure and Appl. Math. 64 (2011), 13051383.

[25] K. Deschout, Multiple orthogonal polynomial ensembles, Ph.D. Thesis, KU Leuven, 2012, https://lirias.kuleuven.be/bitstream/123456789/333369/1/Final+online+version.pdf.

[26] M. Duits and D. Geudens, A critical phenomenon in the two-matrix model in the quartic/quadratic case, arXiv:1111.2162.

[27] M. Duits, D. Geudens and A.B.J. Kuijlaars, A vector equilibrium problem for the two-matrix model in the quartic/quadratic case, Nonlinearity 24 (2011), no. 3, 951993.

[28] M. Duits, A.B.J. Kuijlaars and M. Y. Mo, The Hermitian two-matrix model with an even quartic potential, Mem. Amer. Math. Soc. 217 (2012), no. 1022, vi+105 pp.

[29] N.M. Ercolani and K.T.-R. McLaughlin, Asymptotics and integrable structures for biorthogonal polynomials associated to a random two-matrix model, Physica D 152/153 (2001), 232-268.

[30] B. Eynard and M.L. Mehta, Matrices coupled in a chain: eigenvalue correlations, J. Phys. A 31 (1998), 4449-4456.

[31] P. Ferrari and B. Vető, Non-colliding Brownian bridges and the asymmetric tacnode process, Electron. J. Probab. 17 (2012), no. 44, 1-17.

[32] A. Hardy and A.B.J. Kuijlaars, Weakly admissible vector equilibrium problems, J. Approx. Theory 164 (2012), 854-868.

[33] S.P. Hastings and J.B. McLeod, A boundary value problem associated with the second Painleve transcendent and the Korteweg-de Vries equation, Arch. Rational Mech. Anal. 73 (1980), 31-51. 
[34] K. Johansson, Random matrices and determinantal processes, Mathematical statistical physics, Elsevier B. V., Amsterdam, (2006), 1-55.

[35] K. Johansson, Non-colliding Brownian Motions and the extended tacnode process, arXiv:1105.4027.

[36] M.L. Mehta and P. Shukla, Two coupled matrices: eigenvalue correlations and spacing functions, J. Phys. A 27 (1994), 7793-7803.

[37] A. Okounkov and N. Reshetikhin, Random skew plane partitions and the Pearcey process, Comm. Math. Phys. 269 (2007), no.3, 571-609.

[38] T. Pearcey, The structure of an electromagnetic field in the neighborhood of a cusp of a caustic, Philos. Mag. 37 (1946), 311-317.

[39] A. Soshnikov, Determinantal random point fields, Russian Math. Surveys 55 (2000), 923-975.

[40] C. Tracy and H. Widom, The Pearcey process, Comm. Math. Phys 263 (2006), $381-400$. 